\title{
Modeling dynamics of cancer virotherapy with immune response
}

\author{
Salma M. Al-Tuwairqi ${ }^{*}$ (D), Najwa O. Al-Johani ${ }^{1}$ and Eman A. Simbawa
}

\author{
"Correspondence: \\ saltuwairqi@kau.edu.sa \\ 'Mathematics Department, King \\ Abdulaziz University, Jeddah, Saudi \\ Arabia
}

\begin{abstract}
Virotherapy is a therapeutic treatment for cancer. It uses genetically engineered viruses to selectively infect, replicate in, and destroy cancer cells without damaging normal cells. In this paper, we present a modified model to include, within the dynamics of virotherapy, the interaction between uninfected tumor cells and immune response. The model is analyzed qualitatively to produce five equilibrium points. One of these equilibriums demonstrates the effect observed in virotherapy, where the immune system demolishes infected cells as well as viruses. Moreover, the existence and stability of the equilibrium points are established under certain criteria. Numerical simulations are performed to display the agreement with the analytical results. Finally, parameter analysis is carried out to illustrate which parameters in the model affect the outcome of virotherapy.
\end{abstract}

Keywords: Mathematical model; Cancer; Oncolytic viruses; Stability; Immune response

\section{Introduction}

Cancer is a malignant tumor caused by abnormal division in cells. It infects any part of the body, damages normal cells, and in many cases metastasizes to other areas in the body. According to the World Health Organization [1], cancer was responsible for 9.6 million deaths worldwide in 2018. Traditional therapies for cancer such as surgery, chemotherapy, and radiation are considered to have low efficacy and high toxicity for patients. Therefore, efforts for finding new treatments for cancer is a nonstop process. Fortunately, advances in genetic engineering have paved the way for a new treatment called virotherapy, which has been intensely investigated by researchers. This therapy uses genetically engineered viruses to specifically infect, replicate in, and eradicate cancer cells without causing harm to normal cells. Virotherapy seems to be a promising treatment; however, it faces the challenge of escaping immune cells that destroy the viruses before infecting other cancer cells [2].

Several mathematical models have addressed the interaction between cancer cells and oncolytic viruses to gain a broader understanding of the dynamics of virotherapy. Wodarz [3] formulated a basic model of tumor growth under virotherapy treatment. The model illustrates the interaction between three state variables: uninfected tumor cells, infected tumor cells, and free viruses. Later, Bajzer et al. [4] modified this model by introducing a

(c) The Author(s) 2020. This article is licensed under a Creative Commons Attribution 4.0 International License, which permits use sharing, adaptation, distribution and reproduction in any medium or format, as long as you give appropriate credit to the original author(s) and the source, provide a link to the Creative Commons licence, and indicate if changes were made. The images or other third party material in this article are included in the article's Creative Commons licence, unless indicated otherwise in a credit line to the material. If material is not included in the article's Creative Commons licence and your intended use is not permitted by statutory regulation or exceeds the permitted use, you will need to obtain permission directly from the copyright holder. To view a copy of this licence, visit http://creativecommons.org/licenses/by/4.0/. 
generalized logistic growth of tumor cells, as well as incorporating the elimination of free viruses due to the infection of tumor cells. Also, their model describes the direct infection of uninfected cells from infected cells. However, Tian's model in [5] neglected the direct infection term between uninfected and infected cells and focused on explicitly presenting the parameter of virus replicability known as burst size. The analysis of Tian's model suggests that at large values of the burst size, tumor cells may decrease. Moreover, Ikawati et al. [6] modified the model in [5] by replacing the bilinear incident rate with a standard incident rate because the infection rate depends on the number of cells available at the time of infection.

Other mathematical models have incorporated the immune response since it is one of the major obstacles that hinder the effectiveness of viral therapy. In therapy, the presence of viruses stimulates immune cells against cancer cells due to virus replications within tumor cells, since these viruses are recognized by the immune system as a foreign body. Therefore, immunity not only destroys infected cancer cells but also leads to the clearance of viruses, which counterintuitively minimizes therapy efficiency [2, 7]. Several studies address this side effect of the immune response. One of the early studies that investigated the impact of immune response on tumor-virus dynamics was done by Wodarz [8]. He proposed three models that describe the interactions between uninfected and infected tumor cells with three immune responses: virus-specific Cytotoxic T Lymphocytes (CTL), tumor-specific CTL, and both virus and tumor-specific CTL responses. Later, Ashyani et al. [9] presented a detailed mathematical analysis with simulations of the last model in [8] after modifying it by considering immune responses to both virus and tumor as one variable in the model. The state variables of the models in $[8,9]$ are uninfected tumor cells, infected tumor cells, and immune cells. Phan and Tian [10] added another state variable to them, which describes the virus-free population. They examined the impact of innate immune response on infected cancer cells and the virus population. They concluded from the analysis that when the burst size is large the dynamics of the model is similar to [5] without innate immunity. As for small values of the burst size, the dynamics with innate immunity produce more equilibria. The stability of the outcome of virotherapy in all previous models was investigated analytically and numerically, presenting different criteria for tumor eradication, partial tumor reduction, and therapy failure. Moreover, these models consider the change of cell densities regarding time only. Spatiotemporal dynamics of tumor cells under virotherapy and radiovirotherapy treatments can be found in $[11,12]$, where partial differential equations are involved. Also, more general biological models involving the diffusion of cell densities are presented in [13-15].

In this paper, we modify the mathematical model in [10] by introducing a more realistic interaction between the innate immune system and uninfected tumor cells. This is based on the fact that both tumor and virus-infected cells are recognized by natural killer cells that are part of the innate immune system [16-20]. Therefore, we incorporate in the model terms describing the stimulation of immune response due to the presence of tumors as well as the destruction of tumors due to the release of cytokines from natural killer cells. The aim of this work is to understand the complex interplay among tumor cells, oncolytic viruses, and immune response. Thus, dynamical analysis is employed to investigate the optimal therapeutic strategies for cancer remission.

The paper is organized as follows. In Sect. 2, we formulate the model in a nondimensionalized form. Then we analyze the model qualitatively in Sect. 3 by presenting the existence 
and stability criteria for equilibrium points. Moreover, in Sect. 4 we support the analytical results with numerical experiments as well as illustrate numerically parameter sensitivity of the model. Finally, a brief conclusion is given in Sect. 5 .

\section{Mathematical model}

The model describes the interaction between cancer cells, viral therapy, and immune response. When the patient's body is injected with the virus, the virus infects some of the cancer cells that are then divided into uninfected and infected cells. The population of the model consists of the densities within four groups: uninfected cancer cells $x(t)$, infected cancer cells $y(t)$, free virus $v(t)$, and immune cells $z(t)$. Figure 1 shows a diagram of the interaction between the different population densities in the model. We assume that uninfected cells grow logistically with growth rate $\lambda$, and carrying capacity $C$, and die at a rate $d$. The death of uninfected tumor cells is considered as a new addition to the model in [10], thus, the average life span of the uninfected cancer cells is given by $1 / d$. Moreover, the oncolytic virus infects the cancer cells at a rate $\beta$; as a result, the infected cells lyse at a rate $\delta$. Due to lysis, new virion particles are released with burst size $b$. These free virus particles decay at a rate $\gamma$.

In this model, we consider the role of immune response and its impact on both cancer cells and free viruses. In [10] the model describes the effect of immune cells on the infected cells and free viruses. However, here we incorporate the effect of immune response on both uninfected and infected cancer cells as well as its impact on viruses. Therefore, we assume that the immune cells kill both types of cancer cells: the uninfected and infected cells at rates $\alpha$ and $\mu$, respectively. Also, the immune cells destroy free viruses at a rate $k$. The existence of both the uninfected and infected cells stimulates the immune response at rates $s_{2}$ and $s_{1}$, respectively. Finally, the immune response decays at a rate $\rho$. Therefore, the new parameters in our model are $\alpha, d$, and $s_{2}$.

The dynamics of the model are governed by the following system of nonlinear ordinary differential equations, where all the parameters in the system are nonnegative:

$$
\begin{aligned}
& \frac{d x}{d t}=\lambda x\left(1-\frac{x+y}{C}\right)-\beta x v-\alpha x z-d x, \\
& \frac{d y}{d t}=\beta x v-\mu y z-\delta y,
\end{aligned}
$$

Figure 1 Compartmental diagram for the interaction between different populations for model (1). $x(t)$ and $y(t)$ are the densities of uninfected and infected tumor cell populations, respectively. $v(t)$ is the density of free virus population. $z(t)$ is the density of immune cells population. The new terms are shown in red

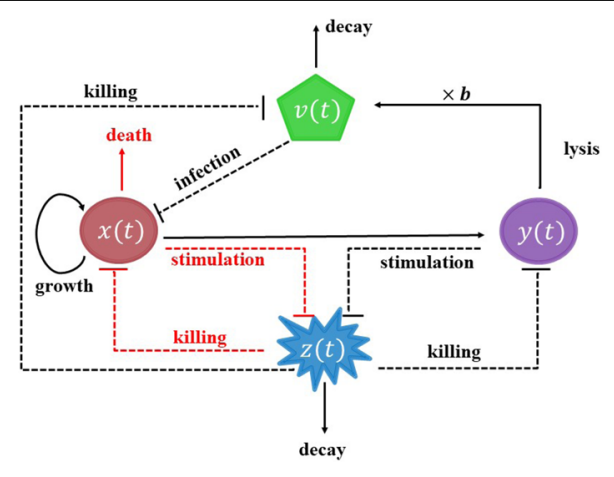




$$
\begin{aligned}
& \frac{d v}{d t}=b \delta y-\beta x v-k v z-\gamma v, \\
& \frac{d z}{d t}=s_{1} y z+s_{2} x z-\rho z
\end{aligned}
$$

with initial conditions

$$
x(0)=x_{0} \geq 0, \quad y(0)=y_{0} \geq 0, \quad v(0)=v_{0} \geq 0, \quad z(0)=z_{0} \geq 0 .
$$

We nondimensionalize the model to reduce the number of its parameters by setting

$$
t=\frac{1}{\delta} \tau, \quad x=C \bar{x}, \quad y=C \bar{y}, \quad v=C \bar{v}, \quad z=C \bar{z},
$$

and renaming the parameters

$$
\begin{aligned}
& r_{1}=\frac{\lambda}{\delta}, \quad a=\frac{\beta C}{\delta}, \quad h_{1}=\frac{\alpha C}{\delta}, \quad d_{1}=\frac{d}{\delta}, \quad c=\frac{\mu C}{\delta}, \\
& h_{2}=\frac{k C}{\delta}, \quad e=\frac{\gamma}{\delta}, \quad m_{1}=\frac{s_{1} C}{\delta}, \quad m_{2}=\frac{s_{2} C}{\delta}, \quad n=\frac{\rho}{\delta} \text {. }
\end{aligned}
$$

Substituting the above in system (1), we obtain

$$
\begin{aligned}
& \frac{d x}{d t}=r_{1} x(1-(x+y))-a x v-h_{1} x z-d_{1} x, \\
& \frac{d y}{d t}=a x v-c y z-y, \\
& \frac{d v}{d t}=b y-a x v-h_{2} v z-e v, \\
& \frac{d z}{d t}=m_{1} y z+m_{2} x z-n z .
\end{aligned}
$$

Also, the initial conditions become

$$
x(0)=\frac{x_{0}}{C}, \quad y(0)=\frac{y_{0}}{C}, \quad v(0)=\frac{\nu_{0}}{C}, \quad z(0)=\frac{z_{0}}{C} .
$$

Note that for simplicity we removed all the bars and write $\tau$ as $t$.

\section{Qualitative analysis}

In this section, we study model (2) qualitatively. First, we determine the positive and bounded region for the state variables, then we obtain the steady-state solutions of the model and examine their stability. The main results are given in the following subsections.

\subsection{Positivity and boundedness}

Theorem 1 All the solutions of system (2) are nonnegative and bounded in some region $\Gamma$ subject to nonnegative initial conditions in $\Gamma$.

Proof Rewrite the first equation of model (2) as

$$
\frac{d x}{x}=\phi_{1}(x, y, v, z) d t
$$


where

$$
\phi_{1}(x, y, v, z)=r_{1}(1-(x+y))-a v-h_{1} z-d_{1} \text {. }
$$

By integrating over $[0, t]$, we can obtain

$$
x(t)=x(0) \exp \left[\int_{0}^{t} \phi_{1}(x, y, v, z) d s\right] .
$$

Since $x(0) \geq 0$, we have $x(t) \geq 0$ for all $t \geq 0$.

From the second equation in (2), we note that

$$
\frac{d y}{d t} \geq-(c z+1) y
$$

then it can be written as

$$
\frac{d y}{y} \geq \phi_{2}(y, z) d t
$$

where

$$
\phi_{2}(y, z)=-(c z+1) .
$$

Integration over $[0, t]$ gives

$$
y(t) \geq y(0) \exp \left[\int_{0}^{t} \phi_{2}(y, z) d s\right] .
$$

If $y(0) \geq 0$, then we have $y(t) \geq 0$ for all $t \geq 0$.

Similarly, the third equation in (2) may be written as

$$
\frac{d v}{v} \geq \phi_{3}(x, z) d t
$$

where

$$
\phi_{3}(x, z)=-\left(a x+h_{2} z+e\right) .
$$

Thus,

$$
v(t) \geq v(0) \exp \left[\int_{0}^{t} \phi_{3}(x, z) d s\right] .
$$

Again, if $v(0) \geq 0$, then we have $v(t) \geq 0$ for all $t \geq 0$.

Finally, we rewrite the last equation in (2) as

$$
\frac{d z}{z}=\phi_{4}(x, y) d t
$$


where

$$
\phi_{4}(x, y)=\left(m_{1} y+m_{2} x-n\right)
$$

Therefore,

$$
z(t)=z(0) \exp \left[\int_{0}^{t} \phi_{4}(x, y) d s\right] .
$$

Also, since $z(0) \geq 0$, we have $z(t) \geq 0$ for all $t \geq 0$. Hence, the solution $(x(t), y(t), v(t), z(t))$ is nonnegative for nonnegative initial data.

Next, we prove that all solutions will remain bounded. We start by rewriting the first equation in (2) as follows:

$$
\frac{d x}{d t} \leq r_{1} x(1-x)
$$

Now, let us consider the differential equation $\frac{d U}{d t}=r_{1} U(1-U)$ with the initial condition $U(0)=U_{0}$. It is possible to check that it has the following solution:

$$
U(t)=\frac{U_{0}}{U_{0}+\left(1-U_{0}\right) e^{-r_{1} t}} .
$$

Hence, $\lim _{t \rightarrow \infty} \sup U(t)=1$.

We know that $\frac{d x}{d t} \leq \frac{d U}{d t}$. Thus, $\lim _{t \rightarrow \infty} \sup x(t) \leq \lim _{t \rightarrow \infty} \sup U(t)$. Therefore,

$$
\lim _{t \rightarrow \infty} \sup x(t) \leq 1
$$

Also, we note that

$$
\begin{aligned}
\frac{d x}{d t}+\frac{d y}{d t} & =r_{1} x(1-(x+y))-h_{1} x z-d_{1} x-c y z-y \\
& \leq r_{1} x(1-(x+y)) \\
& \leq r_{1}(1-(x+y))
\end{aligned}
$$

which gives

$$
\lim _{t \rightarrow \infty} \sup (x(t)+y(t)) \leq 1
$$

Again, by rewriting the third equation in (2) as

$$
\begin{aligned}
\frac{d v}{d t} & \leq b y-e v \\
& \leq b-e v
\end{aligned}
$$

we obtain

$$
\lim _{t \rightarrow \infty} \sup v(t) \leq \frac{b}{e} .
$$


Let

$$
w(t)=x(t)+y(t)+\frac{h}{m} z(t)
$$

where $h=\min \left(h_{1}, c\right), m=\max \left(m_{1}, m_{2}\right)$. Then

$$
\begin{aligned}
\frac{d w}{d t} & =\frac{d x}{d t}+\frac{d y}{d t}+\frac{h}{m} \frac{d z}{d t} \\
& =r_{1} x(1-(x+y))-h_{1} x z-d_{1} x-c y z-y+\frac{h}{m} m_{1} y z+\frac{h}{m} m_{2} x z-\frac{h}{m} n z \\
& \leq r_{1} x-d_{1} x-y-\frac{h}{m} n z \\
& \leq r_{1}-\sigma\left(x+y+\frac{h}{m} z\right),
\end{aligned}
$$

where $\sigma=\min \left(d_{1}, 1, n\right)$. Thus,

$$
\lim _{t \rightarrow \infty} \sup w(t) \leq \frac{r_{1}}{\sigma}
$$

Hence, all the solutions of model (2) are nonnegative and bounded in the following region:

$$
\Gamma=\left\{(x, y, v, z) \in \mathbb{R}_{+}^{4} \mid x \leq 1, x+y \leq 1, v \leq \frac{b}{e}, x+y+\frac{h}{m} z \leq \frac{r_{1}}{\sigma}\right\} .
$$

$\Gamma$ is positively invariant. This means that every solution with initial conditions in $\Gamma$ remains there for all $t>0$, that is, model (2) is well posed.

\subsection{Equilibrium points and basic reproduction number}

The equilibrium points of the system are the steady-state solutions obtained by setting the rates of the equations in (2) to zero. We obtain the following five equilibrium points $E_{i}=\left(x_{i}, y_{i}, v_{i}, z_{i}\right)$, where $i=0,1,2,3,4$ :

$$
\begin{aligned}
& E_{0}=(0,0,0,0), \\
& E_{1}=\left(1-\frac{d_{1}}{r_{1}}, 0,0,0\right), \\
& E_{2}=\left(\frac{e}{a(b-1)}, \frac{e}{a(b-1)}\left(\frac{a(b-1)\left(r_{1}-d_{1}\right)-e r_{1}}{r_{1} e+a(b-1)}\right), \frac{a(b-1)\left(r_{1}-d_{1}\right)-e r_{1}}{a\left(r_{1} e+a(b-1)\right)}, 0\right), \\
& E_{3}=\left(\frac{n}{m_{2}}, 0,0, \frac{r_{1} m_{2}-\left(r_{1} n+d_{1} m_{2}\right)}{h_{1} m_{2}}\right), \\
& E_{4}=\left(x_{4}, y_{4}, v_{4}, z_{4}\right),
\end{aligned}
$$

where $E_{4}$ satisfies the following equations:

$$
\begin{aligned}
& r_{1}\left(1-\left(x_{4}+y_{4}\right)\right)-a v_{4}-h_{1} z_{4}-d_{1}=0, \\
& y_{4}=\frac{a x_{4} v_{4}}{c z_{4}+1},
\end{aligned}
$$




$$
\begin{aligned}
& v_{4}=\frac{b y_{4}}{a x_{4}+h_{2} z_{4}+e}, \\
& m_{1} y_{4}+m_{2} x_{4}-n=0 .
\end{aligned}
$$

The equilibrium points $E_{0}, E_{1}$, and $E_{2}$ are similar to those in [10] when $d_{1}=0 . E_{0}$ represents a free equilibrium where all cancer cells, immune cells, and viruses tend to zero. $E_{1}$ represents a case of no success in treatment when uninfected tumor cells prevail with no existence of any other cells or viruses. However, $E_{2}$ denotes a partial victory for virotherapy treatment where there is coexistence of uninfected and infected cells as well as viruses as described in the equilibrium. The new equilibrium point that is different from the equilibria in [10] is $E_{3}$, where both the infected cells and free viruses tend to zero. This effect is observed in virotherapy [2] where the immune system responds quickly to the presence of viruses and infected cells in the body; as a result, the immune cells destroy the infected cells as well as viruses. As for $E_{4}$, the coexistence of all cells and viruses is present.

Basic reproduction number. Here, we consider the basic reproduction number as the number of secondary cases of infection generated from a single virus in a population where all tumor cells are susceptible to infection. Let $\mathcal{P}=(y, x, v, z)$. Then model $(2)$ can be rewritten as $\mathcal{P}^{\prime}=\mathcal{F}(\mathcal{P})-\mathcal{V}(\mathcal{P})$, where

$$
\mathcal{F}(\mathcal{P})=\left[\begin{array}{c}
a x v \\
0 \\
0 \\
0
\end{array}\right]
$$

and

$$
\mathcal{V}(\mathcal{P})=\left[\begin{array}{c}
c y z+y \\
-r_{1} x(1-(x+y))+a x v+h_{1} x z+d_{1} x \\
-b y+a x v+h_{2} v z+e v \\
-m_{1} y z-m_{2} x z+n z
\end{array}\right] .
$$

Now, evaluating the Jacobian of $\mathcal{F}$ at $E_{1}$, we have

$$
D\left(\mathcal{F}\left(E_{1}\right)\right)=\left[\begin{array}{cccc}
0 & 0 & a\left(1-\frac{d_{1}}{r_{1}}\right) & 0 \\
0 & 0 & 0 & 0 \\
0 & 0 & 0 & 0 \\
0 & 0 & 0 & 0
\end{array}\right]
$$

Also,

$$
D\left(\mathcal{V}\left(E_{1}\right)\right)=\left[\begin{array}{cccc}
1 & 0 & 0 & 0 \\
r_{1}-d_{1} & r_{1}-d_{1} & a\left(1-\frac{d_{1}}{r_{1}}\right) & h_{1}\left(1-\frac{d_{1}}{r_{1}}\right) \\
-b & 0 & a\left(1-\frac{d_{1}}{r_{1}}\right)+e & 0 \\
0 & 0 & 0 & -m_{2}\left(1-\frac{d_{1}}{r_{1}}\right)+n
\end{array}\right]
$$


Let $F$ and $V$ be as follows:

$$
F=\left[\begin{array}{ccc}
0 & 0 & a\left(1-\frac{d_{1}}{r_{1}}\right) \\
0 & 0 & 0 \\
0 & 0 & 0
\end{array}\right],
$$

and

$$
V=\left[\begin{array}{ccc}
1 & 0 & 0 \\
r_{1}-d_{1} & r_{1}-d_{1} & a\left(1-\frac{d_{1}}{r_{1}}\right) \\
-b & 0 & a\left(1-\frac{d_{1}}{r_{1}}\right)+e
\end{array}\right] .
$$

By calculating the next generation matrix [21], we obtain

$$
F V^{-1}=\left[\begin{array}{ccc}
\frac{a b\left(r_{1}-d_{1}\right)}{a\left(r_{1}-d_{1}\right)+e r_{1}} & 0 & \frac{-a\left(r_{1}-d_{1}\right)}{a\left(d_{1}-r_{1}\right)-e r_{1}} \\
0 & 0 & 0 \\
0 & 0 & 0
\end{array}\right] .
$$

Then, the basic reproduction number $R_{0}$ is given by

$$
R_{0}=\rho\left(F V^{-1}\right)=\frac{a b\left(r_{1}-d_{1}\right)}{a\left(r_{1}-d_{1}\right)+e r_{1}},
$$

where $\rho\left(F V^{-1}\right)$ denotes the spectral radius of a matrix $F V^{-1}$.

The equilibrium points have biological meaning, therefore all of its components must be nonnegative and exist in $\Gamma$. One can see that $E_{0}$ exists always, whereas $E_{1}$ exists only if $r_{1}>d_{1}$. On the other hand, if the conditions $b>1$ and $R_{0}>1$ are satisfied, then $E_{2}$ exists. As for $E_{3}$, its existence condition is $\frac{m_{2}\left(r_{1}-d_{1}\right)}{n r_{1}}>1$.

\subsection{Stability of equilibria}

We investigate the local stability of the equilibrium points by using the linearization method [22]. The method, in brief, is to evaluate the Jacobian matrix of the nonlinear system at an equilibrium point and then prove that the eigenvalues of the matrix of the linearized system are negative. Moreover, we examine the global stability of the equilibrium points using Lyapunov functions [23].

Local stability

Theorem 2 The equilibrium point $E_{0}$ is locally asymptotically stable if $r_{1}<d_{1}$.

Proof The Jacobian matrix of system (2) at $E_{0}$ gives

$$
J\left(E_{0}\right)=\left[\begin{array}{cccc}
r_{1}-d_{1} & 0 & 0 & 0 \\
0 & -1 & 0 & 0 \\
0 & b & -e & 0 \\
0 & 0 & 0 & -n
\end{array}\right] .
$$

Thus, the eigenvalues of $J\left(E_{0}\right)$ are

$$
\lambda_{1}=r_{1}-d_{1}, \quad \lambda_{2}=-1, \quad \lambda_{3}=-e, \quad \lambda_{4}=-n .
$$


Clearly, all eigenvalues are negative and $\lambda_{1}$ is negative if $r_{1}<d_{1}$. Hence, $E_{0}$ is locally asymptotically stable if $r_{1}<d_{1}$.

Theorem 3 The equilibrium point $E_{1}$ is locally asymptotically stable if $\frac{m_{2}\left(r_{1}-d_{1}\right)}{n r_{1}}<1$ and $R_{0}<1$.

Proof By evaluating the Jacobian matrix of system (2) at $E_{1}$, we get

$$
J\left(E_{1}\right)=\left[\begin{array}{cccc}
d_{1}-r_{1} & d_{1}-r_{1} & -a\left(1-\frac{d_{1}}{r_{1}}\right) & -h_{1}\left(1-\frac{d_{1}}{r_{1}}\right) \\
0 & -1 & a\left(1-\frac{d_{1}}{r_{1}}\right) & 0 \\
0 & b & -a\left(1-\frac{d_{1}}{r_{1}}\right)-e & 0 \\
0 & 0 & 0 & m_{2}\left(1-\frac{d_{1}}{r_{1}}\right)-n
\end{array}\right]
$$

Solving the characteristic equation $\left|J\left(E_{1}\right)-\lambda I\right|=0$, we obtain the eigenvalues: $\lambda_{1}=$ $m_{2}\left(1-d_{1} / r_{1}\right)-n, \lambda_{2}=d_{1}-r_{1}$, and $\lambda_{3,4}$ satisfy the equation

$$
\lambda^{2}+a_{1} \lambda+a_{2}=0
$$

where

$$
\begin{aligned}
& a_{1}=a\left(1-\frac{d_{1}}{r_{1}}\right)+e+1, \\
& a_{2}=a\left(1-\frac{d_{1}}{r_{1}}\right)+e-b a\left(1-\frac{d_{1}}{r_{1}}\right) .
\end{aligned}
$$

It is obvious that $\lambda_{1}$ is negative if $\frac{m_{2}\left(r_{1}-d_{1}\right)}{n r_{1}}<1$ and $\lambda_{2}$ is always negative. By using RouthHurwitz criteria [22], the eigenvalues $\lambda_{3,4}$ are negative if $a_{1}>0$ and $a_{2}>0$. Clearly $a_{1}$ is positive. As for $a_{2}$, we have

$$
\begin{aligned}
a_{2} & =a\left(1-\frac{d_{1}}{r_{1}}\right)+e-b a\left(1-\frac{d_{1}}{r_{1}}\right) \\
& =a\left(1-\frac{d_{1}}{r_{1}}\right)(1-b)+e \\
& =\frac{a}{r_{1}}\left(r_{1}-d_{1}\right)(1-b)+e \\
& =\frac{a}{r_{1}}\left(r_{1}-d_{1}\right)-\frac{a b}{r_{1}}\left(r_{1}-d_{1}\right)+e .
\end{aligned}
$$

Thus, for $a_{2}$ to be positive we must have $a b\left(r_{1}-d_{1}\right)<a\left(r_{1}-d_{1}\right)+r_{1} e$, that is, $R_{0}<1$. Hence, $E_{1}$ is locally asymptotically stable if $\frac{m_{2}\left(r_{1}-d_{1}\right)}{n r_{1}}<1$ and $R_{0}<1$.

Theorem 4 The equilibrium point $E_{2}$ is locally asymptotically stable if $m_{1} y_{2}+m_{2} x_{2}<n$ and $r_{1}>a$. 
Proof The Jacobian matrix of system (2) at $E_{2}$ is

$$
J\left(E_{2}\right)=\left[\begin{array}{cccc}
-r_{1} x_{2} & -r_{1} x_{2} & -a x_{2} & -h_{1} x_{2} \\
a v_{2} & -1 & a x_{2} & -c y_{2} \\
-a v_{2} & b & -a x_{2}-e & -h_{2} v_{2} \\
0 & 0 & 0 & m_{1} y_{2}+m_{2} x_{2}-n
\end{array}\right]
$$

After solving the characteristic equation $\left|J\left(E_{2}\right)-\lambda I\right|=0$, we find one eigenvalue explicitly, $\lambda_{1}=m_{1} y_{2}+m_{2} x_{2}-n$, whereas other eigenvalues $\lambda_{2,3,4}$ satisfy the following equation:

$$
\lambda^{3}+a_{1} \lambda^{2}+a_{2} \lambda+a_{3}=0
$$

where

$$
\begin{aligned}
& a_{1}=r_{1} x_{2}+1+a x_{2}+e, \\
& a_{2}=r_{1} x_{2}\left(1+a x_{2}+e\right)+\left(r_{1}-a\right) y_{2}, \\
& a_{3}=\left(r_{1} e+a(b-1)\right) y_{2} .
\end{aligned}
$$

It is clear that $a_{1}>0$ and $a_{3}>0$ since $E_{2}$ exists if $b>1$. We need to show that $a_{1} a_{2}>a_{3}$, then by using Routh-Hurwitz criteria, we prove that the eigenvalues $\lambda_{2,3,4}$ are negative. Now,

$$
\begin{aligned}
a_{1} a_{2}-a_{3}= & \left(r_{1} x_{2}+1+a x_{2}+e\right)\left(r_{1} x_{2}+a r_{1} x_{2}^{2}+r_{1} e x_{2}\right)+y_{2}\left(\left(r_{1}^{2}-a^{2}\right) x_{2}\right. \\
& \left.+r_{1}-a(e+b)\right) \\
= & \frac{e}{a(b-1)}\left(\frac{r_{1} e}{a(b-1)}+1+\frac{a e}{a(b-1)}+e\right)\left(r_{1}+\frac{a r_{1} e}{a(b-1)}+r_{1} e\right) \\
& +\frac{y_{2}}{a(b-1)}\left(\left(r_{1}^{2}-a^{2}\right) e+r_{1} a(b-1)-a^{2}(b-1)(e+b)\right) \\
= & \frac{e}{a(b-1)}\left(\frac{r_{1} e}{a(b-1)}+1+\frac{a e}{a(b-1)}+e\right)\left(r_{1}+\frac{a r_{1} e}{a(b-1)}+r_{1} e\right) \\
& +\frac{y_{2}}{a(b-1)}\left(e\left(r_{1}^{2}-a^{2} b\right)+a(b-1)\left(r_{1}-a b\right)\right) .
\end{aligned}
$$

Note that we have used $x_{2}=\frac{e}{a(b-1)}$. For $a_{1} a_{2}-a_{3}$ to be positive, we must have $r_{1}>a b$, which leads to the condition $r_{1}>a$ since $b>1$. Also, $\lambda_{1}$ is negative if $m_{1} y_{2}+m_{2} x_{2}<n$. Thus, $E_{2}$ is locally asymptotically stable if $r_{1}>a$ and $m_{1} y_{2}+m_{2} x_{2}<n$.

Theorem 5 The equilibrium point $E_{3}$ is locally asymptotically stable if

$$
\frac{\left(c z_{3}+1\right)\left(a x_{3}+h_{2} z_{3}+e\right)}{a b x_{3}}>1 .
$$


Proof Evaluating the Jacobian matrix of system (2) at $E_{3}$, we get

$$
J\left(E_{3}\right)=\left[\begin{array}{cccc}
-r_{1} x_{3} & -r_{1} x_{3} & -a x_{3} & -h_{1} x_{3} \\
0 & -c z_{3}-1 & a x_{3} & 0 \\
0 & b & -a x_{3}-h_{2} z_{3}-e & 0 \\
m_{2} z_{3} & m_{1} z_{3} & 0 & 0
\end{array}\right]
$$

The characteristic equation $\left|J\left(E_{3}\right)-\lambda I\right|=0$ gives

$$
\left(\lambda^{2}+a_{1} \lambda+a_{2}\right)\left(\lambda^{2}+b_{1} \lambda+b_{2}\right)=0,
$$

where

$$
\begin{aligned}
& a_{1}=a x_{3}+h_{2} z_{3}+e+c z_{3}+1, \\
& a_{2}=c a x_{3} z_{3}+a x_{3}-a b x_{3}+c h_{2} z_{3}^{2}+h_{2} z_{3}+c e z_{3}+e, \\
& b_{1}=r_{1} x_{3}, \\
& b_{2}=m_{2} h_{1} x_{3} z_{3} .
\end{aligned}
$$

Then, by Routh-Hurwitz criteria, the characteristic equation has negative roots if $a_{1}>0$, $a_{2}>0, b_{1}>0$, and $b_{2}>0$. Clearly, $a_{1}, b_{1}$, and $b_{2}$ are positive, while $a_{2}>0$ if

$$
\begin{aligned}
& c a x_{3} z_{3}+a x_{3}+c h_{2} z_{3}^{2}+h_{2} z_{3}+c e z_{3}+e>a b x_{3}, \\
& c z_{3}\left(a x_{3}+e\right)+a x_{3}+e+h_{2} z_{3}\left(c z_{3}+1\right)>a b x_{3}, \\
& \left(a x_{3}+e\right)\left(c z_{3}+1\right)+h_{2} z_{3}\left(c z_{3}+1\right)>a b x_{3}, \\
& \frac{\left(c z_{3}+1\right)\left(a x_{3}+h_{2} z_{3}+e\right)}{a b x_{3}}>1 .
\end{aligned}
$$

Thus, $E_{3}$ is locally asymptotically stable if $\frac{\left(c z_{3}+1\right)\left(a x_{3}+h_{2} z_{3}+e\right)}{a b x_{3}}>1$.

Theorem 6 The equilibrium point $E_{4}$ is locally asymptotically stable provided $a_{i}>0$, where $i=1,2,3,4$, and $a_{1} a_{2} a_{3}>a_{3}^{2}+a_{1}^{2} a_{4}$. Here $a_{1}, a_{2}, a_{3}$, and $a_{4}$ are provided in the proof.

Proof Evaluating the Jacobian at the equilibrium point $E_{4}$ and solving the characteristic equation $\left|J\left(E_{4}\right)-\lambda I\right|=0$, we obtain the equation

$$
\lambda^{4}+a_{1} \lambda^{3}+a_{2} \lambda^{2}+a_{3} \lambda+a_{4}=0
$$

where

$$
\begin{aligned}
a_{1}= & \left(a+r_{1}\right) x_{4}+\left(h_{2}+c\right) z_{4}+e+1, \\
a_{2}= & \left(r_{1} x_{4}+c z_{4}+1\right)\left(a x_{4}+h_{2} z_{4}+e\right)+r_{1} x_{4}\left(c z_{4}+1+a v_{4}\right)-a b x_{4}+c m_{1} y_{4} z_{4} \\
& +h_{1} m_{2} x_{4} z_{4}-a^{2} x_{4} v_{4},
\end{aligned}
$$




$$
\begin{aligned}
a_{3}= & r_{1} x_{4}\left(c z_{4}+1+a v_{4}\right)\left(a x_{4}+h_{2} z_{4}+e\right)-a b x_{4}\left(r_{1} x_{4}-a v_{4}\right)+a m_{1} h_{2} x_{4} v_{4} z_{4} \\
& +\left(a x_{4}+h_{2} z_{4}+e\right)\left(c m_{1} y_{4} z_{4}+h_{1} m_{2} x_{4} z_{4}\right)+r_{1} c x_{4} y_{4} z_{4}\left(m_{1}-m_{2}\right)+h_{1} a m_{1} x_{4} v_{4} z_{4} \\
& +h_{1} c m_{2} x_{4}\left(z_{4}\right)^{2}+h_{1} m_{2} x_{4} z_{4}-a^{2} x_{4} v_{4}\left(r_{1} x_{4}+c z_{4}+1\right)-a h_{2} m_{2} x_{4} v_{4} z_{4}, \\
a_{4}= & a x_{4}\left(r_{1} x_{4}-a v_{4}\right)\left(m_{1} h_{2} v_{4} z_{4}\right)-a h_{2} m_{2} x_{4} v_{4} z_{4}\left(r_{1} x_{4}+c z_{4}+1\right) \\
& +\left(a x_{4}+h_{2} z_{4}+e\right)\left(r_{1} c x_{4} y_{4} z_{4}\left(m_{1}-m_{2}\right)+h_{1} a m_{1} x_{4} v_{4} z_{4}\right. \\
& \left.+h_{1} c m_{2} x_{4}\left(z_{4}\right)^{2}+h_{1} m_{2} x_{4} z_{4}\right)-\left(a m_{1} v_{4} z_{4}+b m_{2} z_{4}\right)\left(a x_{4}\left(c y_{4}+h_{1} x_{4}\right)\right) .
\end{aligned}
$$

Clearly, $a_{1}>0$. Hence, by the Routh-Hurwitz criterion, the local asymptotic stability of $E_{4}$ is guaranteed under the conditions stated in the theorem.

Remark 7 Note that if the equilibrium point $E_{0}$ is stable, then $E_{1}$ does not exist. Similarly, if $E_{1}$ exists, then $E_{0}$ is unstable. Also, if $E_{1}$ is stable, then neither $E_{2}$ nor $E_{3}$ exists and vice versa.

\section{Global stability}

Theorem 8 The equilibrium point $E_{0}$ is globally asymptotically stable if $r_{1}<d_{1}$ and $b<1$.

Proof Define the Lyapunov function as

$$
L(x, y, v, z)=\frac{m_{2} c}{m_{1} h_{1}} x+y+v+\frac{c}{m_{1}} z .
$$

Clearly, $L$ is a positive definite function. Computing the derivative of $L$ along the solutions of model (2), we get

$$
\begin{aligned}
L^{\prime}= & \frac{m_{2} c}{m_{1} h_{1}}\left(r_{1} x(1-(x+y))-a x y-h_{1} x z-d_{1} x\right)+a x v-c y z-y \\
& +b y-a x v-h_{2} v z-e v+\frac{c}{m_{1}}\left(m_{1} y z+m_{2} x z_{n} z\right) .
\end{aligned}
$$

Collecting terms, we have

$$
\begin{aligned}
L^{\prime}= & \frac{m_{2} c}{m_{1} h_{1}}\left(r_{1}-d_{1}\right) x-\frac{m_{2} c}{m_{1} h_{1}} r_{1} x^{2}-\frac{m_{2} c}{m_{1} h_{1}} r_{1} x y-\frac{m_{2} c}{m_{1} h_{1}} a x v \\
& +x z\left(-\frac{m_{2} c}{m_{1}}+m_{2} \frac{c}{m_{1}}\right)+y z\left(-c+m_{1} \frac{c}{m_{1}}\right)+(b-1) y-h_{2} v z-e v-\frac{n c}{m_{1}} z \\
\leq & \frac{m_{2} c}{m_{1} h_{1}}\left(r_{1}-d_{1}\right) x+(b-1) y-e v-\frac{n c}{m_{1}} z .
\end{aligned}
$$

If $r_{1}<d_{1}$ and $b<1$, then $L^{\prime} \leq 0$ and $L^{\prime}=0$ if and only if $x=y=v=z=0$. Thus, the largest invariant set in $\left\{(x, y, v, z) \in \Gamma: L^{\prime}=0\right\}$ is $E_{0}$. Hence, by La Salle's invariance principle [23], $E_{0}$ is globally asymptotically stable.

Theorem 9 The equilibrium point $E_{1}$ is globally stable if $1-\frac{e r_{1}}{a}<b<1$ and $R_{1}<1$, where $R_{1}=\frac{h_{1} m_{1}(1-b)}{n c r_{1} x_{1}^{2}}\left(x_{1}+\frac{c m_{2} r_{1} x_{1}^{2}}{4 m_{1} h_{1}(1-b)}\right)^{2}$. 
Proof Define the positive definite Lyapunov function

$$
L(x, y, v, z)=\frac{2(1-b)}{r_{1} x_{1}^{2}}\left(x-x_{1}\right)^{2}+y+v+\frac{c}{m_{1}} z .
$$

By differentiating $L$ and using $d_{1}=r_{1}\left(1-x_{1}\right)$, we obtain

$$
\begin{aligned}
L^{\prime}= & \frac{4(1-b)}{r_{1} x_{1}^{2}}\left(x-x_{1}\right) x^{\prime}+y^{\prime}+v^{\prime}+\frac{c}{m_{1}} z^{\prime} \\
= & \frac{4(1-b)}{r_{1} x_{1}^{2}}\left(x-x_{1}\right)\left[-r_{1} x^{2}-r_{1} x y-a x v-h_{1} x z+r_{1} x x_{1}\right] \\
& +a x v-c y z-y+b y-a x v-h_{2} v z-e v+\frac{c}{m_{1}}\left(m_{1} y z+m_{2} x z-n z\right) \\
= & \frac{4(1-b)}{r_{1} x_{1}^{2}}\left(-r_{1} x\left(x-x_{1}\right)^{2}-\left(r_{1} y+a v\right)\left(x-\frac{x_{1}}{2}\right)^{2}+\left(r_{1} y+a v\right) \frac{x_{1}^{2}}{4}-h_{1} x^{2} z+h_{1} x_{1} x z\right) \\
& +(b-1) y-h_{2} v z-e v+\frac{c}{m_{1}}\left(m_{2} x z-n z\right) .
\end{aligned}
$$

Collecting and simplifying terms, we get

$$
\begin{aligned}
L^{\prime} \leq & y\left(b-1+\frac{4(1-b)}{r_{1} x_{1}^{2}} \frac{r_{1} x_{1}^{2}}{4}\right)+v\left(\frac{4(1-b)}{r_{1} x_{1}^{2}} \frac{a x_{1}^{2}}{4}-e\right) \\
& +z\left(-\frac{4(1-b)}{r_{1} x_{1}^{2}} h_{1} x^{2}+\left(\frac{4(1-b)}{r_{1} x_{1}^{2}} h_{1} x_{1}+\frac{c m_{2}}{m_{1}}\right) x-\frac{c n}{m_{1}}\right) \\
= & v\left(\frac{a(1-b)}{r_{1}}-e\right)-\frac{4(1-b)}{r_{1} x_{1}^{2}} h_{1} z\left(x-\frac{1}{2}\left(x_{1}+\frac{c m_{2} r_{1} x_{1}^{2}}{4 m_{1} h_{1}(1-b)}\right)\right)^{2} \\
& +\frac{(1-b)}{r_{1} x_{1}^{2}} h_{1}\left(x_{1}+\frac{c m_{2} r_{1} x_{1}^{2}}{4 m_{1} h_{1}(1-b)}\right)^{2}-\frac{c n}{m_{1}} \\
\leq & v\left(\frac{a(1-b)}{r_{1}}-e\right)+\frac{(1-b)}{r_{1} x_{1}^{2}} h_{1}\left(x_{1}+\frac{c m_{2} r_{1} x_{1}^{2}}{4 m_{1} h_{1}(1-b)}\right)^{2}-\frac{c n}{m_{1}} .
\end{aligned}
$$

If $1-\frac{e r_{1}}{a}<b<1$ and $R_{1}<1$, then $E_{1}$ is globally stable.

Theorem 10 The equilibrium point $E_{2}$ is globally stable if $m_{2}$ cax $_{2}<h_{1}$ em $_{1}, 1+$ $\frac{r_{1}\left(r_{1} e+a(b-1)\right)}{a\left(r_{1}-d_{1}\right)+e r_{1}}<R_{0}<1+\frac{r_{1} e\left(r_{1} e+a(b-1)\right)}{a b\left(a\left(r_{1}-d_{1}\right)+e r_{1}\right)}, 1<b<\frac{r_{1} e}{a}$, and $R_{2}<1$, where $R_{2}=\frac{v_{2} m_{1}}{c n}\left(h_{2}+\frac{c e}{b-1}\right)+$ $\underline{h_{1} m_{1} e}$.

Proof Define the Lyapunov function as follows:

$$
L(x, y, v, z)=\frac{e}{a x_{2}}\left(x-x_{2}-x_{2} \ln \frac{x}{x_{2}}\right)+y-y_{2}-y_{2} \ln \frac{y}{y_{2}}+v-v_{2}-v_{2} \ln \frac{v}{v_{2}}+\frac{c}{m_{1}} z .
$$

It is simple to show that $L$ is a positive definite function, since the function $f(u)=u-1-$ $\ln (u)$ is positive for all $u>0$. Evaluating $L^{\prime}$, we have

$$
\begin{aligned}
L^{\prime}= & \frac{e}{a x_{2}}\left(1-\frac{x_{2}}{x}\right)\left[r_{1} x(1-(x+y))-a x v-h_{1} x z-d_{1} x\right]+\left(1-\frac{y_{2}}{y}\right)[a x v-c y z-y] \\
& +\left(1-\frac{v_{2}}{v}\right)\left[b y-a x v-h_{2} v z-e v\right]+\frac{c}{m_{1}}\left(m_{1} y z+m_{2} x z-n z\right) .
\end{aligned}
$$


Since $E_{2}$ is an equilibrium point to system (2), then

$$
\begin{aligned}
& d_{1}=\frac{1}{x_{2}}\left(r_{1} x_{2}\left(1-\left(x_{2}+y_{2}\right)\right)-a x_{2} v_{2}\right), \\
& 0=-a x_{2} v_{2}+y_{2} \\
& e=\frac{1}{v_{2}}\left(b y_{2}-a x_{2} v_{2}\right) .
\end{aligned}
$$

Substituting (4)-(6) into (3), we get

$$
\begin{aligned}
L^{\prime}= & \frac{e}{a x_{2}}\left(1-\frac{x_{2}}{x}\right)\left[-r_{1} x x_{2}\left(\frac{x}{x_{2}}-1\right)-r_{1} x_{2} y_{2}\left(\frac{x y}{x_{2} y_{2}}-\frac{x}{x_{2}}\right)\right. \\
& \left.-a x_{2} v_{2}\left(\frac{x v}{x_{2} v_{2}}-\frac{x}{x_{2}}\right)-h_{1} x z\right] \\
& +\left(1-\frac{y_{2}}{y}\right)\left[-a x_{2} v_{2}\left(1-\frac{x v}{x_{2} v_{2}}\right)-c y z-y_{2}\left(\frac{y}{y_{2}}-1\right)\right] \\
& +\left(1-\frac{v_{2}}{v}\right)\left[-b y_{2}\left(\frac{v}{v_{2}}-\frac{y}{y_{2}}\right)\right. \\
& \left.-a x_{2} v_{2}\left(\frac{x v}{x_{2} v_{2}}-\frac{v}{v_{2}}\right)-h_{2} v z\right]+\frac{c}{m_{1}}\left(m_{1} y z+m_{2} x z-n z\right) .
\end{aligned}
$$

Collecting and simplifying terms yields

$$
\begin{aligned}
L^{\prime}= & -\frac{e}{a} r_{1} x\left(1-\frac{x_{2}}{x}\right)\left(\frac{x}{x_{2}}-1\right)+\frac{x}{x_{2}}\left[\frac{e}{a}\left(r_{1} y_{2}+a v_{2}\right)+a x_{2} v_{2}\right]+\frac{y}{y_{2}}\left[\frac{e}{a} r_{1} y_{2}+b y_{2}\right] \\
& -\frac{e}{a} r_{1} y_{2} \frac{x y}{x_{2} y_{2}}-\frac{e}{a} r_{1} y_{2}+\frac{v}{v_{2}}\left[e v_{2}+a x_{2} v_{2}-b y_{2}\right]+\frac{x v}{x_{2} v_{2}}\left[-e v_{2}+a x_{2} v_{2}-a x_{2} v_{2}\right] \\
& -e v_{2}+\frac{e}{a x_{2}} h_{1}\left(-x z+x_{2} z\right)-a x_{2} v_{2}+\frac{y_{2}}{y} a x_{2} v_{2}-\frac{x v y_{2}}{x_{2} v_{2} y} a x_{2} v_{2} \\
& -c y z+c y_{2} z-y_{2}\left(1-\frac{y_{2}}{y}\right)\left(\frac{y}{y_{2}}-1\right) \\
& +b y_{2}-\frac{v_{2} y}{v y_{2}} b y_{2}-a x_{2} v_{2}-h_{2} v z+h_{2} v_{2} z+\frac{c}{m_{1}}\left(m_{1} y z+m_{2} x z-n z\right) .
\end{aligned}
$$

From the inequality of arithmetic and geometric means, we have

$$
\left(1-\frac{x_{2}}{x}\right)\left(\frac{x}{x_{2}}-1\right)=\frac{x}{x_{2}}+\frac{x_{2}}{x}-2>0 .
$$

Employing this and recollecting terms in (7), we obtain

$$
\begin{aligned}
L^{\prime} \leq & \frac{x}{x_{2}}\left[\frac{e}{a}\left(r_{1} y_{2}+a v_{2}\right)+a x_{2} v_{2}\right]+\frac{y}{y_{2}}\left[\frac{e}{a} r_{1} y_{2}+b y_{2}\right] \\
& -\frac{e}{a} r_{1} y_{2}+b y_{2}+\frac{v}{v_{2}}\left[e v_{2}+a x_{2} v_{2}-b y_{2}\right]
\end{aligned}
$$




$$
\begin{aligned}
& +x z\left(-\frac{e}{a x_{2}} h_{1}+\frac{c}{m_{1}} m_{2}\right)+z\left(\frac{e}{a} h_{1}+c y_{2}+h_{2} v_{2}-\frac{c}{m_{1}} n\right)-a x_{2} v_{2}\left(1-\frac{y_{2}}{y}\right) \\
& -y_{2}\left(1-\frac{y_{2}}{y}\right)\left(\frac{y}{y_{2}}-1\right)-a x_{2} v_{2} .
\end{aligned}
$$

Using (5) and (6) and simplifying, we get

$$
\begin{aligned}
L^{\prime} \leq & \frac{x}{x_{2}}\left[\frac{e}{a x_{2}} r_{1} x_{2} y_{2}+b y_{2}\right]+\frac{y}{y_{2}}\left[\frac{e}{a x_{2}} r_{1} x_{2} y_{2}+b y_{2}\right] \\
& +y_{2}\left(b-\frac{e}{a} r_{1}\right)+x z\left(-\frac{e}{a x_{2}} h_{1}+\frac{c}{m_{1}} m_{2}\right) \\
& +z\left(\frac{e}{a} h_{1}+c \frac{e}{b-1} v_{2}+h_{2} v_{2}-\frac{c}{m_{1}} n\right) .
\end{aligned}
$$

From (4), we have

$$
\begin{aligned}
\frac{e}{a x_{2}} r_{1} x_{2} y_{2}+b y_{2}= & \frac{e}{a x_{2}}\left[r_{1} x_{2}-r_{1} x_{2}^{2}-a x_{2} v_{2}-d_{1} x_{2}\right]+b y_{2} \\
\leq & \left(\frac{r_{1}}{a}-v_{2}\right) e-\frac{e}{a} r_{1} x_{2}+b y_{2} \\
= & -\frac{a\left(r_{1}-d_{1}\right)+e r_{1}}{a\left(r_{1} e+a(b-1)\right)}\left[-\frac{r_{1}\left(r_{1} e+a(b-1)\right)}{a\left(r_{1}-d_{1}\right)+e r_{1}}+R_{0}-1\right] \\
& -\frac{b x_{2}\left(a\left(r_{1}-d_{1}\right)+e r_{1}\right)}{r_{1} e+a(b-1)}\left[-R_{0}+1+\frac{e r_{1}\left(r_{1} e+a(b-1)\right)}{a b\left(a\left(r_{1}-d_{1}\right)+e r_{1}\right)}\right] .
\end{aligned}
$$

If $m_{2} c a x_{2}<h_{1} e m_{1}, 1+\frac{r_{1}\left(r_{1} e+a(b-1)\right)}{a\left(r_{1}-d_{1}\right)+e r_{1}}<R_{0}<1+\frac{r_{1} e\left(r_{1} e+a(b-1)\right)}{a b\left(a\left(r_{1}-d_{1}\right)+e r_{1}\right)}, 1<b<\frac{r_{1} e}{a}$, and $R_{2}=\frac{v_{2} m_{1}}{c n}\left(h_{2}+\right.$ $\left.\frac{c e}{b-1}\right)+\frac{h_{1} m_{1} e}{a c n}<1$, then $L^{\prime} \leq 0$. Thus, $E_{2}$ is globally stable.

Theorem 11 The equilibrium point $E_{3}$ is globally stable if $b<1$ and $\frac{h_{1} m_{1}}{n}>\frac{r_{1}}{z_{3}}+\frac{a c}{e}$.

Proof Define the Lyapunov function as follows:

$$
L(x, y, v, z)=\frac{n}{h_{1} x_{3}}\left(x-x_{3}-x_{3} \ln \frac{x}{x_{3}}\right)+\frac{m_{1}}{c} y+\frac{m_{1}}{c} v+z-z_{3}-z_{3} \ln \frac{z}{z_{3}}
$$

Clearly, $L$ is a positive definite function. Differentiating $L$, we get

$$
\begin{aligned}
L^{\prime}= & \frac{n}{h_{1} x_{3}}\left(1-\frac{x_{3}}{x}\right)\left[r_{1} x(1-(x+y))-a x v-h_{1} x z-d_{1} x\right]+\frac{m_{1}}{c}[a x v-c y z-y] \\
& +\frac{m_{1}}{c}\left[b y-a x v-h_{2} v z-e v\right]+\left(1-\frac{z_{3}}{z}\right)\left[m_{1} y z+m_{2} x z-n z\right] .
\end{aligned}
$$

Substituting $d_{1}=\left(r_{1} x_{3}-r_{1} x_{3}^{2}-h_{1} x_{3} z_{3}\right) / x_{3}$ into (9) and simplifying, we have

$$
\begin{aligned}
L^{\prime}= & -\frac{n r_{1}}{h_{1}} x\left(1-\frac{x_{3}}{x}\right)\left(\frac{x}{x_{3}}-1\right)-\frac{n r_{1}}{h_{1} x_{3}} x y+\frac{n r_{1}}{h_{1}} y-\frac{a n}{h_{1} x_{3}} x v+\frac{a n}{h_{1}} v-\frac{n}{x_{3}} x z \\
& +\frac{n}{x_{3}} z_{3} x+n z-n z_{3}+\frac{m_{1}}{c}[a x v-c y z-y]+\frac{m_{1}}{c}\left[b y-a x v-h_{2} v z-e v\right]+m_{1} y z \\
& +m_{2} x z-n z-m_{1} y z_{3}-m_{2} x z_{3}+n z_{3} .
\end{aligned}
$$


Using $x_{3}=n / m_{2}$ and collecting terms, we obtain

$$
\begin{aligned}
L^{\prime} & \leq y\left[\frac{n r_{1}}{h_{1}}+\frac{m_{1}}{c}(b-1)-m_{1} z_{3}\right]+v\left[\frac{a n}{h_{1}}-\frac{m_{1}}{c} e\right]+x\left[\frac{n}{x_{3}} z_{3}-m_{2} z_{3}\right]-m_{2} x z+m_{2} x z \\
& =\frac{n z_{3}}{h_{1}} y\left[\frac{r_{1}}{z_{3}}+\frac{m_{1} h_{1}}{n c z_{3}}(b-1)-\frac{h_{1} m_{1}}{n}\right]+\frac{n e}{h_{1} c} v\left[\frac{a c}{e}-\frac{h_{1} m_{1}}{n}\right] .
\end{aligned}
$$

If $b<1$ and $\frac{h_{1} m_{1}}{n}>\frac{r_{1}}{z_{3}}+\frac{a c}{e}$, then $L^{\prime} \leq 0$. Hence, $E_{3}$ is globally stable.

\section{Numerical analysis}

In this section, we solve system (2) numerically and illustrate different simulations of the model using ode45, a solver in Matlab for ordinary differential equations [27]. Also, we explore the sensitivity of the parameters to gain more insight into those critical parameters that may lead to optimal therapeutic strategies. The values of the parameters are taken from [10, 24-26] and are shown in Table 1. The numerical simulations are demonstrated for the nondimensionalized model (2), where all population classes are divided by the carrying capacity of tumor cells. Therefore, we indicate the classes in the population as relative densities of uninfected cells, infected cells, free virus, and immune cells. Also, we refer to the nondimensionalized time as a relative time where one relative time is equivalent to 18 hours, that is, one apoptotic cycle for infected cells. The values of the nondimensionalized parameters are as follows [10]:

$$
\begin{array}{llll}
r_{1}=0.36, & a=0.1, & h_{1}=0.36, & d_{1}=0.1278, \quad c=0.48, \quad b=2, \\
h_{2}=0.16, & e=0.2, \quad m_{1}=0.6, \quad m_{2}=0.29, \quad n=0.036 .
\end{array}
$$

\subsection{Numerical experiments}

We solve model (2) numerically with the following different initial values:

(a) $x(0)=0.5, y(0)=0, v(0)=0.01, z(0)=0.01$;

\begin{tabular}{|c|c|c|c|c|}
\hline Parameter & Description & Value & Units & Reference \\
\hline$\lambda$ & Tumor growth rate & $2 \times 10^{-2}$ & $1 / \mathrm{h}$ & {$[24]$} \\
\hline$d$ & Death rate of uninfected tumor cells & 0.0071 & $1 / \mathrm{h}$ & [25] \\
\hline$\beta$ & Infection rate of the virus & $7 / 10 \times 10^{-9}$ & $\mathrm{~mm}^{3} / \mathrm{h}$ virus & {$[24]$} \\
\hline$\delta$ & Death rate of infected tumor cells & $1 / 18$ & $1 / \mathrm{h}$ & {$[24]$} \\
\hline$b$ & Burst size of free virus & 50 & viruses/cell & {$[24]$} \\
\hline$\gamma$ & Clearance rate of the virus & 0.0119 & $1 / \mathrm{h}$ & {$[26]$} \\
\hline$\alpha$ & $\begin{array}{l}\text { Immune killing rate of uninfected tumor } \\
\text { cells }\end{array}$ & $2 \times 10^{-8}$ & $\mathrm{~mm}^{3} / \mathrm{h}$ immune cell & estimated \\
\hline$\mu$ & $\begin{array}{l}\text { Immune killing rate of infected tumor } \\
\text { cells }\end{array}$ & $2 \times 10^{-8}$ & $\mathrm{~mm}^{3} / \mathrm{h}$ immune cell & {$[24]$} \\
\hline k & Immune killing rate of the virus & $10^{-8}$ & $\mathrm{~mm}^{3} / \mathrm{h}$ immune cell & [24] \\
\hline$s_{1}$ & $\begin{array}{l}\text { Stimulation rate of the immune response } \\
\text { by infected cells }\end{array}$ & $5.6 \times 10^{-7}$ & $\mathrm{~mm}^{3} / \mathrm{h}$ infected cell & {$[24]$} \\
\hline$s_{2}$ & $\begin{array}{l}\text { Stimulation rate of the immune response } \\
\text { by uninfected cells }\end{array}$ & $5.6 \times 10^{-7}$ & $\mathrm{~mm}^{3} / \mathrm{h}$ uninfected cell & estimated \\
\hline$\rho$ & Clearance rate of the immune response & 0.002 & $1 / \mathrm{h}$ & [10] \\
\hline
\end{tabular}

(b) $x(0)=0.7, y(0)=0, v(0)=0.1, z(0)=0.1$;

(c) $x(0)=0.9, y(0)=0, v(0)=0.5, z(0)=0.2$.

Table 1 The model parameters, their description and values 
(a)

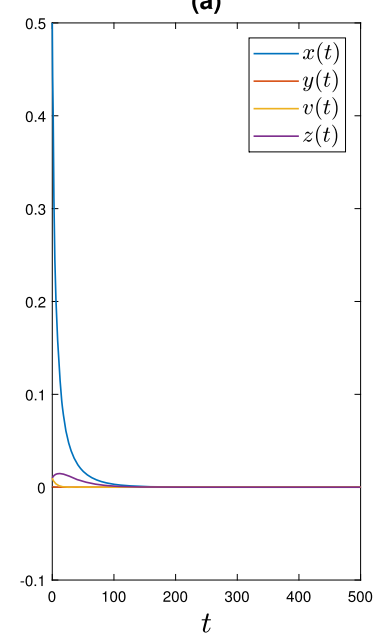

(b)

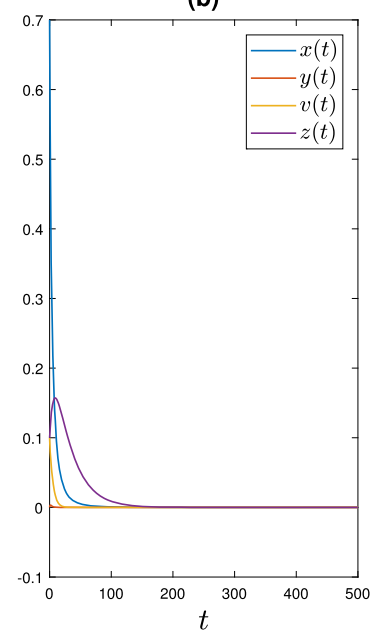

(c)

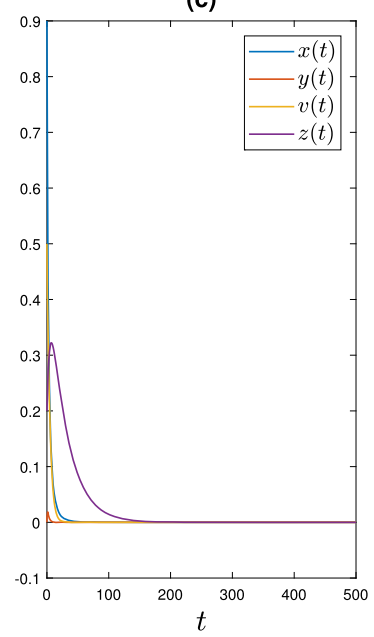

Figure 2 Numerical simulations of model (2) showing the time variation in the size of all relative populations of the model with various initial values. For these simulations, we used the following parameter values: $r_{1}=0.36, a=0.1, h_{1}=0.36, d_{1}=0.39, c=0.48, b=2, h_{2}=0.16, e=0.2, m_{1}=0.6, m_{2}=0.29$, and $n=0.036$

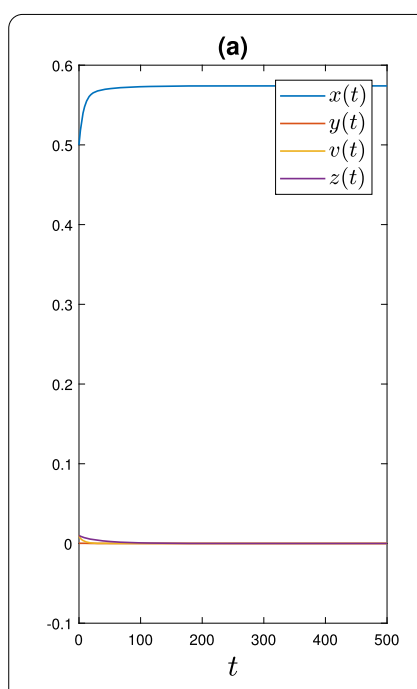

(b)

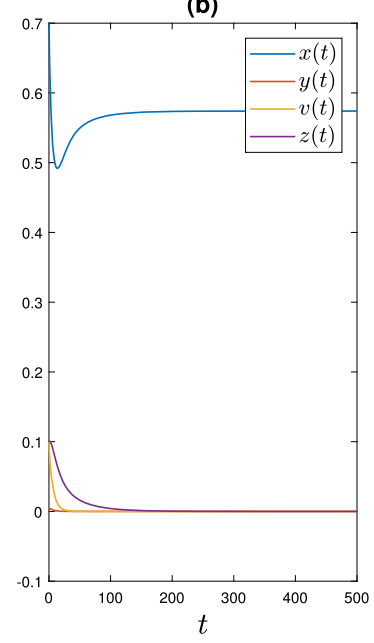

(c)

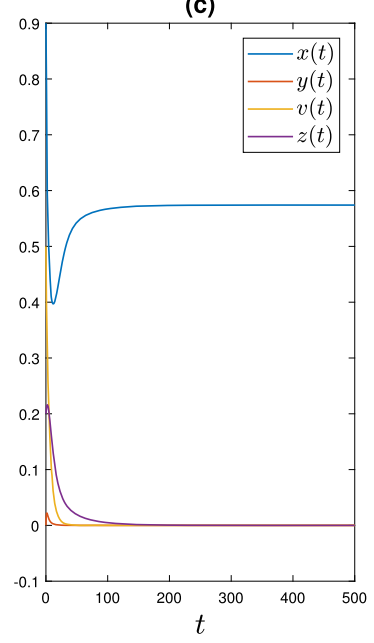

Figure 3 Numerical simulations of model (2) showing the time variation in the size of all relative populations of the model with various initial values. For these simulations, we used the following parameter values: $r_{1}=0.3, a=0.1, h_{1}=0.36, d_{1}=0.1278, c=0.48, b=2, h_{2}=0.16, e=0.2, m_{1}=0.6, m_{2}=0.29$, and $n=0.19$

Also, we set the parameters as in (11); however, we may change certain values of the parameters to satisfy the existence and stability constraints for each equilibrium point. First, we change the relative death rate to the value $d_{1}=0.39$. Figure 2 shows that the solution curves tend to the equilibrium point $E_{0}=(0,0,0,0)$ since its stability condition $r_{1}<d_{1}$ is satisfied. Thus, $E_{0}$ is locally asymptotically stable for different sets of the initial conditions. If we change the parameters $r_{1}$ and $n$ to be $0.3,0.19$, respectively, then the conditions $\frac{m_{2}\left(r_{1}-d_{1}\right)}{n r_{1}}<1$ and $R_{0}<1$ are achieved, and thus, $E_{1}=(0.5740,0,0,0)$ is locally asymptotically stable (see Fig. 3). By setting the parameters at $b=9$ and $n=0.19$, the criteria for the existence and stability of $E_{2}$ are fulfilled. Therefore, Fig. 4 illustrates the local asymptotic stability of $E_{2}=(0.25,0.0326,1.3046,0)$ with different initial val- 
(a)

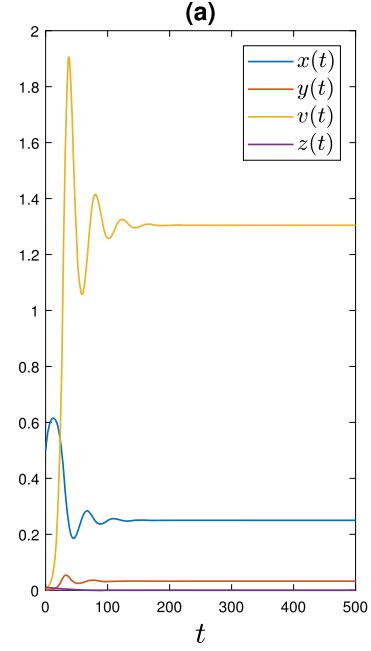

(b)

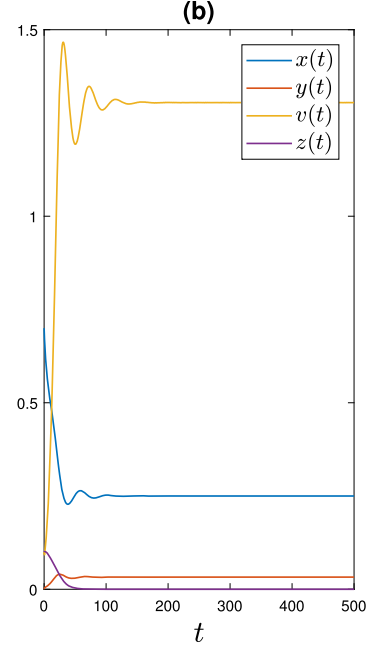

(c)

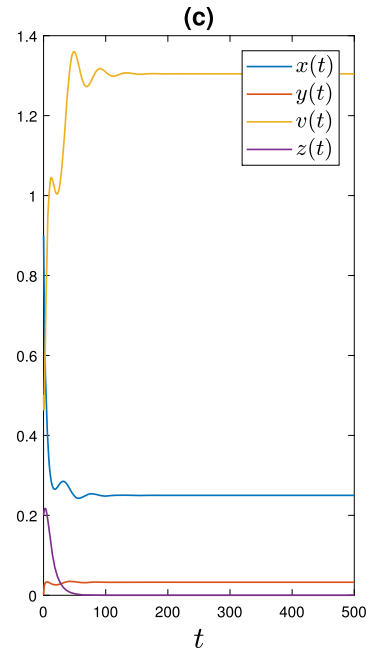

Figure 4 Numerical simulations of model (2) showing the time variation in the size of all relative populations of the model with various initial values. For these simulations, we used the following parameter values: $r_{1}=0.36, a=0.1, h_{1}=0.36, d_{1}=0.1278, c=0.48, b=9, h_{2}=0.16, e=0.2, m_{1}=0.6, m_{2}=0.29$, and $n=0.19$

(a)

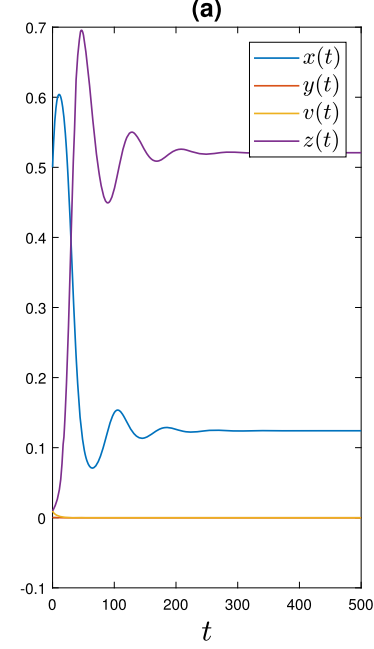

(b)

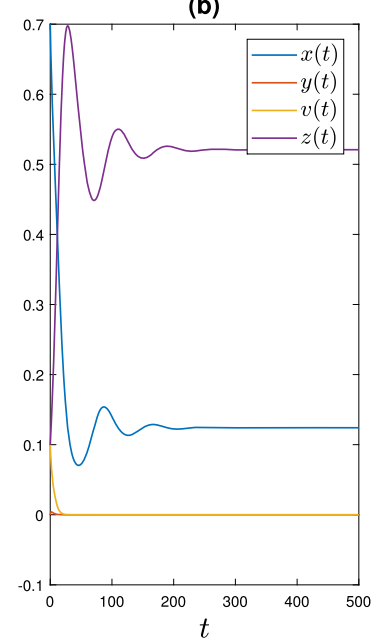

(c)

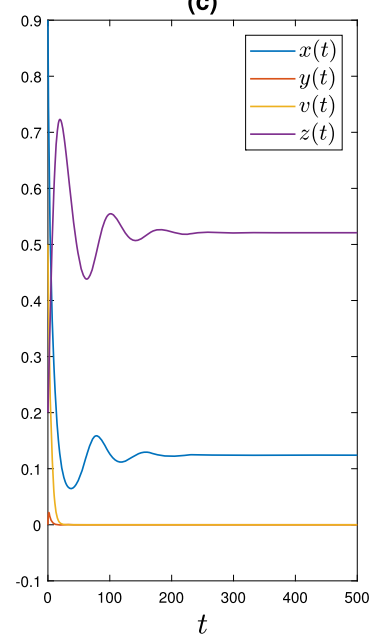

Figure 5 Numerical simulations of model (2) showing the time variation in the size of all relative populations of the model with various initial value. For these simulations, we used the following parameter values: $r_{1}=0.36, a=0.1, h_{1}=0.36, d_{1}=0.1278, c=0.48, b=2, h_{2}=0.16, e=0.2, m_{1}=0.6, m_{2}=0.29$, and $n=0.036$

ues. On the other hand, if we keep the parameters set as in (11), then the conditions for $E_{3}$ are satisfied and $E_{3}=(0.1241,0,0,0.5209)$ is locally asymptotically stable as seen in Fig. 5. Finally, if we change the values of the following parameters: $a=0.3, h_{1}=0.6$, $c=0.3, e=0.1, m_{2}=0.1, n=0.05$, then Fig. 6 shows the local asymptotic stability of $E_{4}=(0.3752,0.0208,0.1878,0.0555)$. Hence, from these experiments we may say that the numerical and qualitative results are in good agreement.

\subsection{Parameter analysis}

In this section, we explore the changes in the dynamics of model (2) by varying two potential parameters for virotherapy: the burst size $(b)$ and the relative clearance rate of viruses 

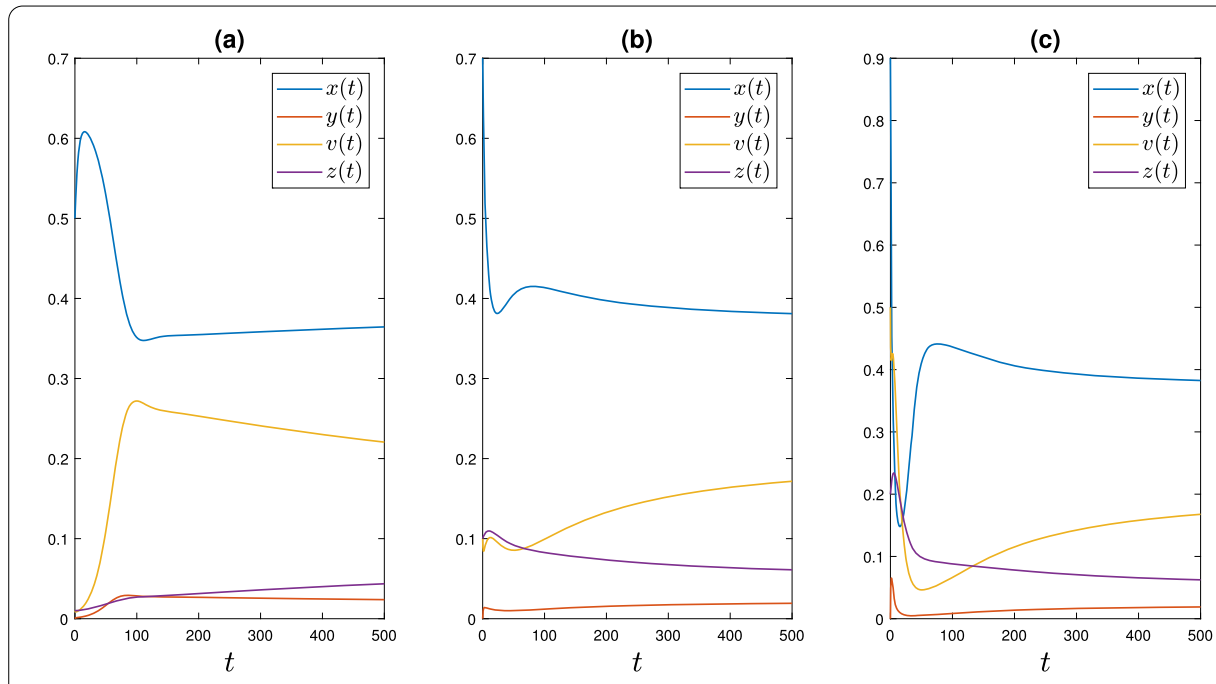

Figure 6 Numerical simulations of model (2) showing the time variation in the size of all relative populations of the model with various initial values. For these simulations, we used the following parameter values: $r_{1}=0.36, a=0.3, h_{1}=0.6, d_{1}=0.1278, c=0.3, b=2, h_{2}=0.16, e=0.1, m_{1}=0.6, m_{2}=0.1$, and $n=0.05$

(e). Also, we examine the effect of varying the new parameters in our model on treatment: that is, the relative immune killing rate of uninfected tumor cells $\left(h_{1}\right)$ and the relative stimulation rate of immune response by uninfected cells $\left(m_{2}\right)$. The numerical simulations are carried out when the relative infection rate is low $(a=0.1)$ and when it is high $(a=0.5)$. All parameters are set as in (11) except for the relative clearance rate of immune response, we set $n=0.16$. Also, we set the initial values to be: $x(0)=0.5, y(0)=0, v(0)=0.01, z(0)=0.01$.

The burst size $(b)$ represents the number of new viruses resulting from lysis of infected cancer cells. Figure 7 shows the time variation in the size of all relative populations. We find that whether the infection rate is low or high when $b$ is small, the model tends to the equilibrium point $E_{3}$. This is probably because there are not enough virions, therefore, the immune cells manage to eliminate all viruses and infected cells. However, when $b$ is large the model approaches $E_{2}$. This means that an increase in $b$ leads to partial success for virotherapy treatment. However, when $b$ increases even more for a high infection rate, oscillations with decay [28] occur in the solution curves of uninfected cells, infected cells, and viruses. This effect is also observed in [10, 24].

Next, we vary $h_{1}$, the relative killing rate of uninfected cancer cells due to immune cells. Figure 8 illustrates the time variations in each compartment of model (2) with low and high infection rates. We find that, for low viral infection, whether the value of $h_{1}$ is small or large, the model tends to the equilibrium $E_{3}$. This may be because the infection rate is small and, thus, the immune cells eradicate infected cells and viruses easily. The larger the value of $h_{1}$ the lesser the time the immune cells take to kill tumor cells. As a result, both tumor and immune cells decrease. On the other hand, for high viral infection, the model approaches $E_{2}$ whether $h_{1}$ is large or small. This reflects the significance of the high infection rate.

As for the relative viral clearance rate $e$, we find in Fig. 9 that whether $e$ takes small or large values, the model tends to the equilibrium $E_{3}$ when the infection rate is low. Similar results are illustrated for the high infection rate when $e$ is large. However, for small values 

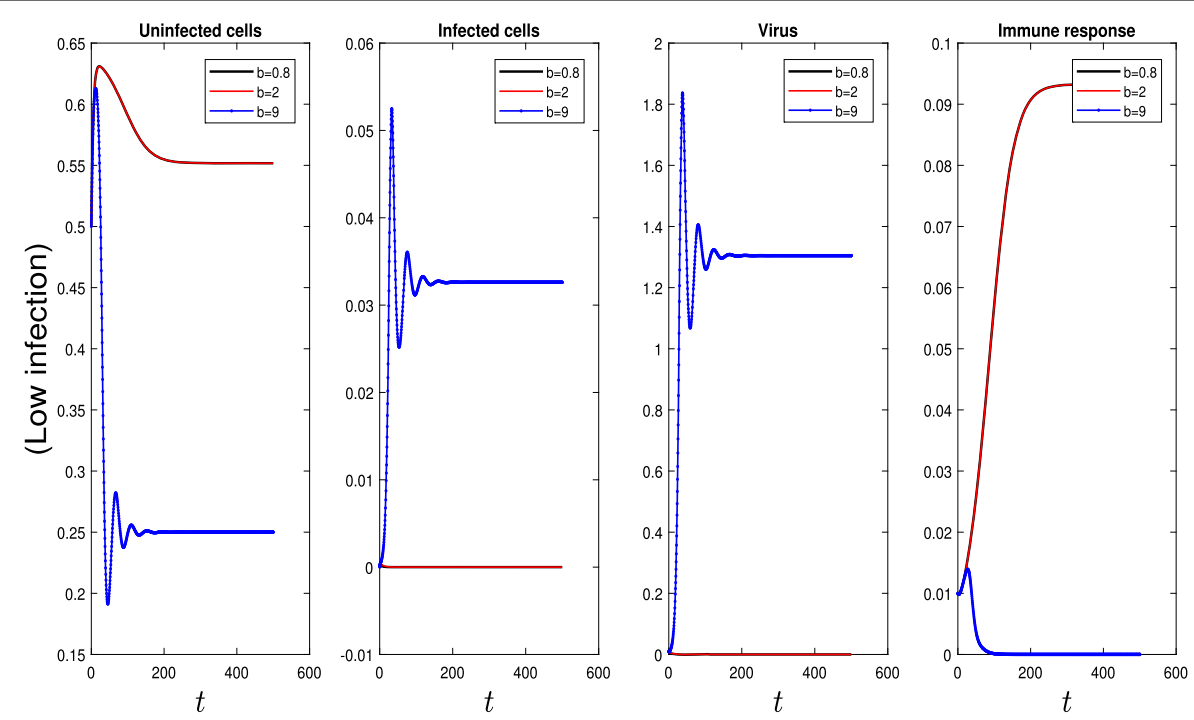

(a)
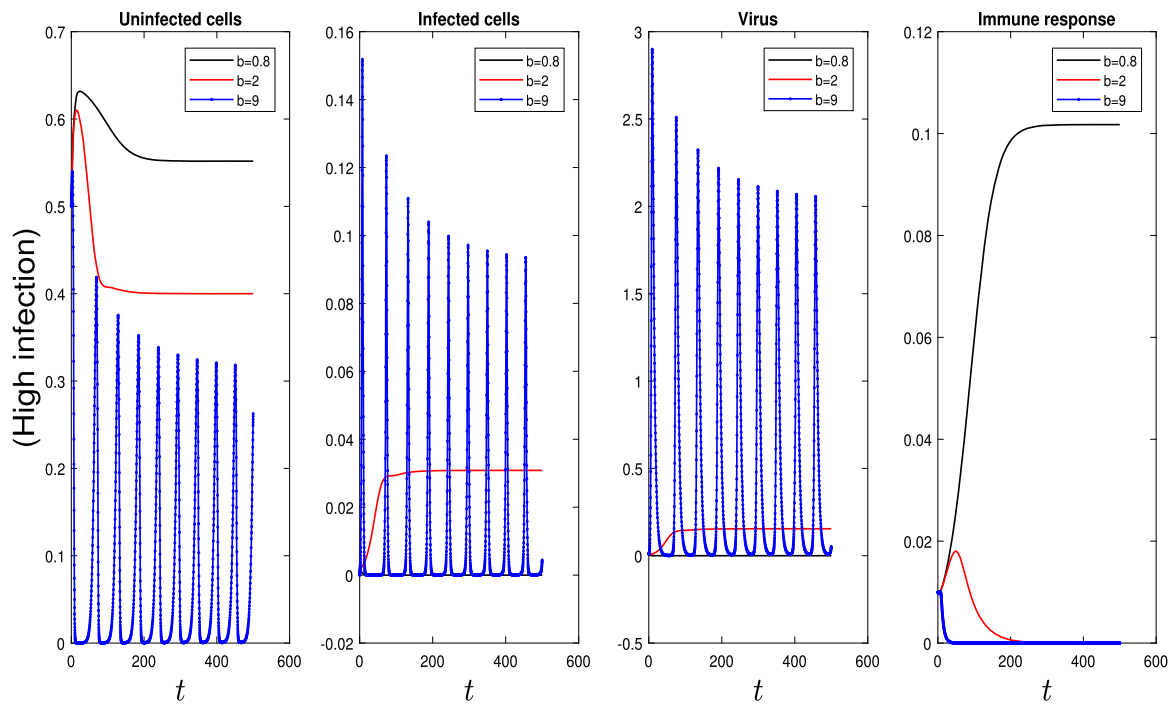

(b)

Figure 7 Numerical simulations of model (2) with initial values: $x(0)=0.5, y(0)=0, v(0)=0.01$, and $z(0)=0.01$, showing the time variation in each compartment of the model with varying values of $b$ as shown in the legend (other parameters are set as in (11) except $n=0.16$ ). The simulations are carried out for two cases of infection rate, (a) low infection ( $a=0.1)$ and (b) high infection $(a=0.5)$

of $e$, the model approaches $E_{2}$. This means that if the viral clearance rate is not small, then no matter what the viral infection rate is, the treatment will not be effective.

Finally, we vary $m_{2}$, the relative rate of stimulating immune response due to uninfected tumor cells. We find that, for low viral infection, the model tends to $E_{3}$. As $m_{2}$ becomes large, the response of immune cells increases; consequently, the size of uninfected tumor cells decreases (see Fig. 10). For high viral infection, the model approaches $E_{3}$ as $m_{2}$ becomes large. However, when $m_{2}$ is small with a high infection rate, the model tends to $E_{2}$. Although $E_{2}$ represents partial success for virotherapy, it is not the case here as shown in Fig. 10. The size of tumor cells is much less when $m_{2}$ is large than when $m_{2}$ is small. Thus, 

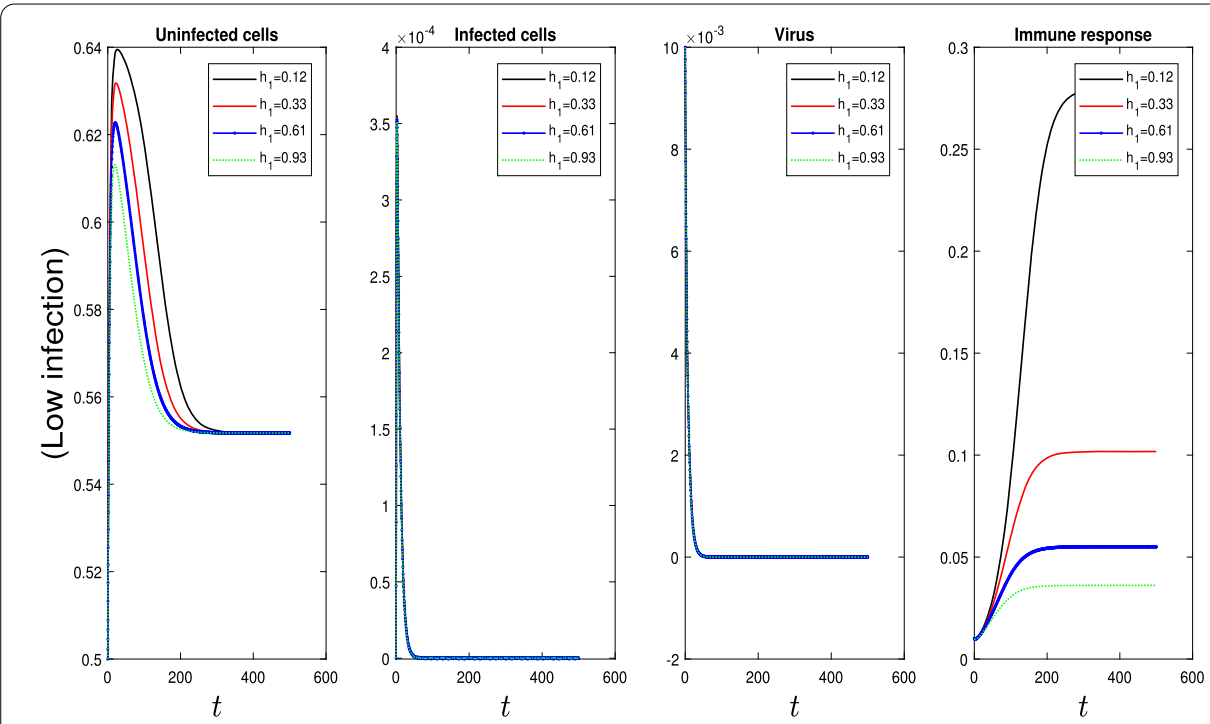

(a)
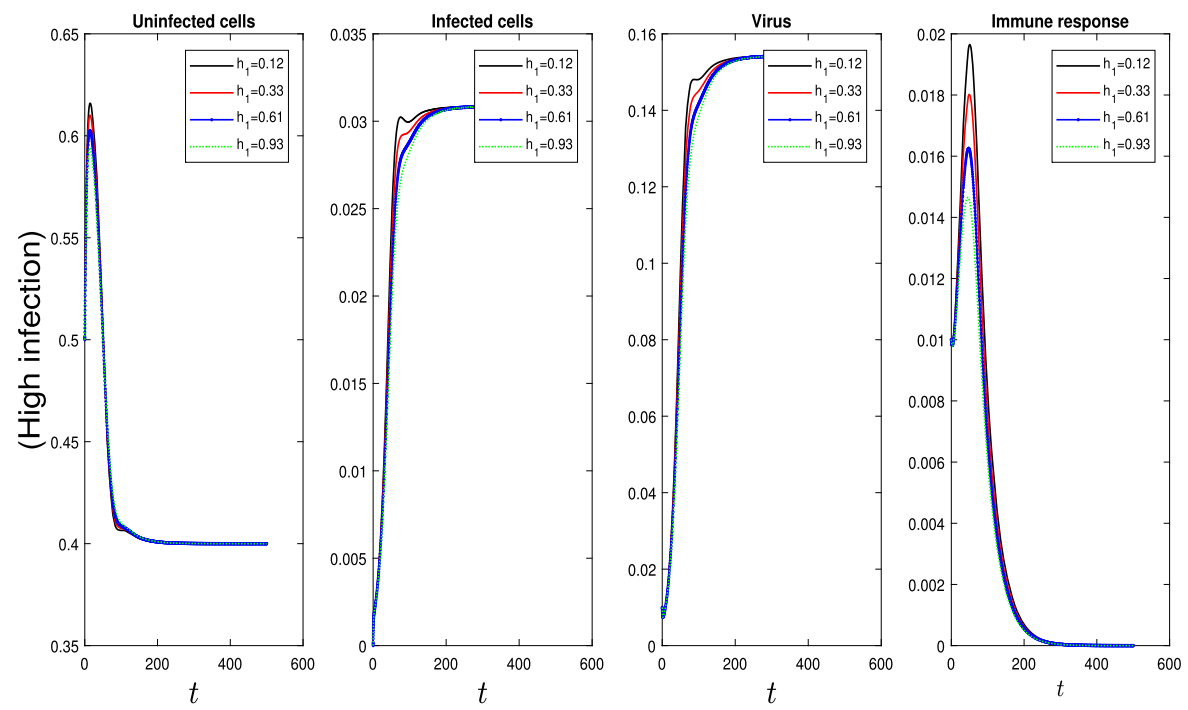

(b)

Figure 8 Numerical simulations of model (2) with initial values: $x(0)=0.5, y(0)=0, v(0)=0.01$, and $z(0)=0.01$, showing the time variation in each compartment of the model with varying values of $h_{1}$ as shown in the legend (other parameters are set as in (11) except $n=0.16$ ). The simulations are carried out for two cases of infection rate, (a) low infection $(a=0.1)$ and $(\mathbf{b})$ high infection $(a=0.5)$

for high viral infection, it is better for the model to reach $E_{3}$ with large values of $m_{2}$ than to reach $E_{2}$ with low values of $m_{2}$.

From the above analysis, we conclude that the high infection rate plays a significant role in controlling tumor size. Alongside with a high infection rate, a low viral clearance rate leads to partial success in treatment, and a high stimulating immune response reduces the size of the tumor. Also, a large burst size, whether the viral infection is high or low, leads to partial success in virotherapy. 

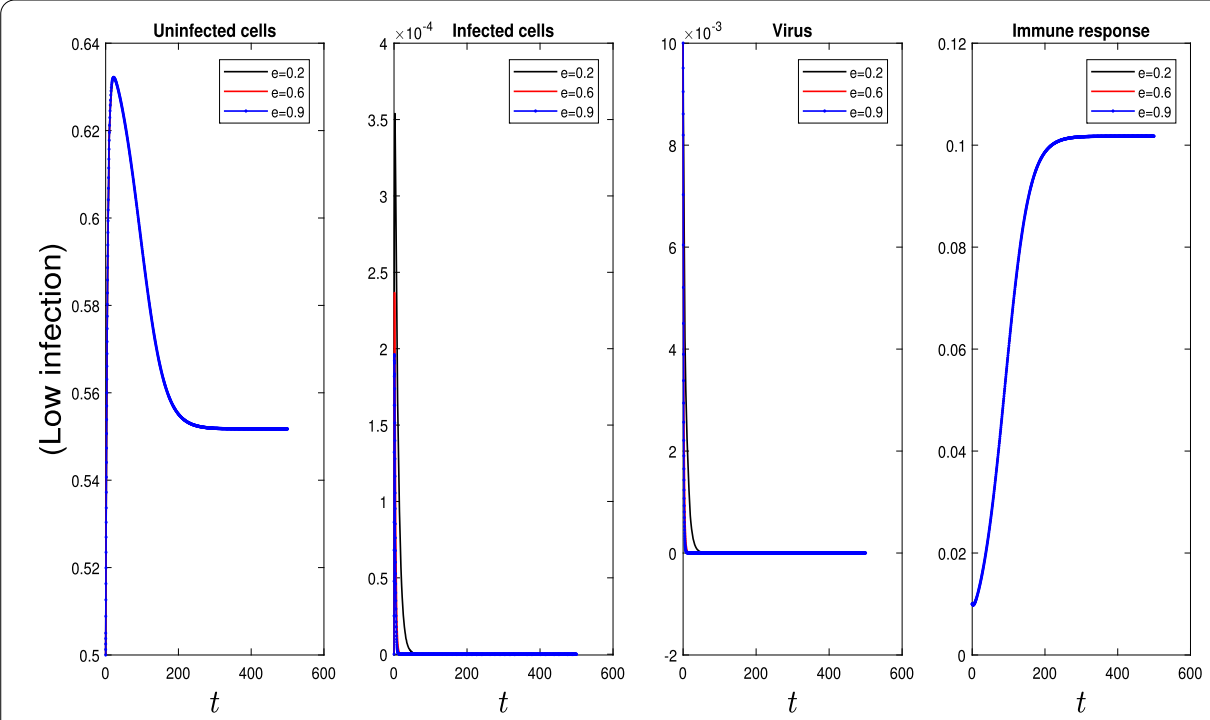

(a)
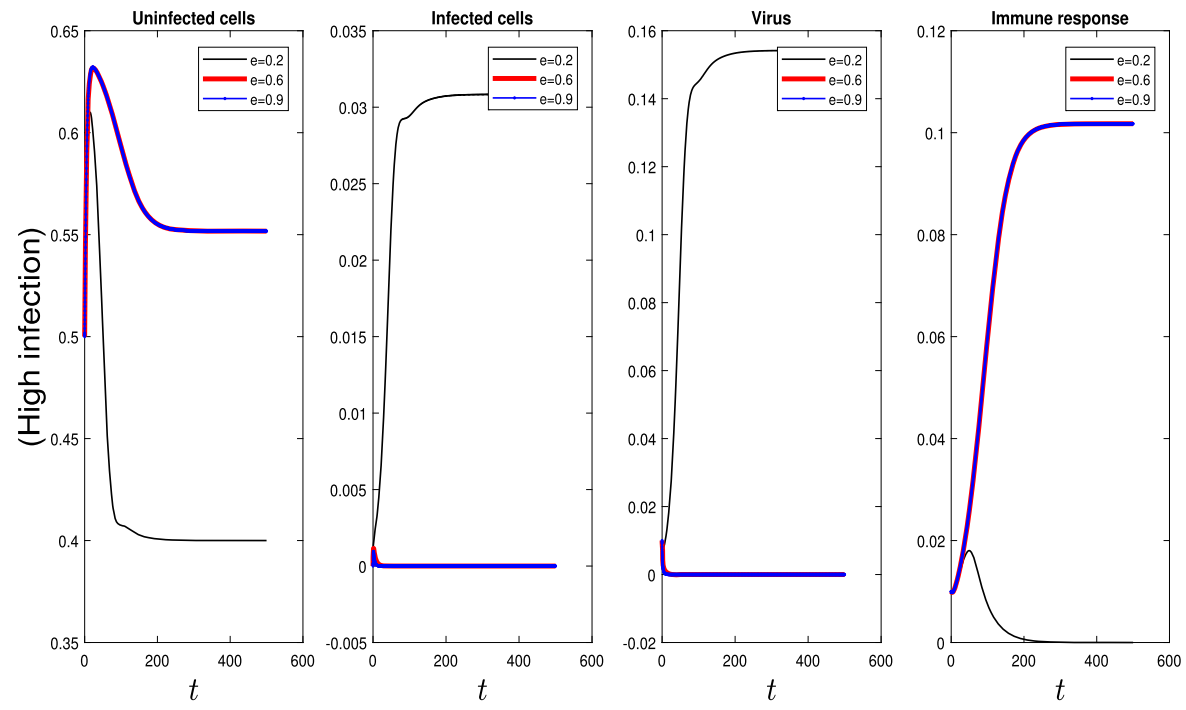

(b)

Figure 9 Numerical simulations of model (2) with initial values: $x(0)=0.5, y(0)=0, v(0)=0.01$, and $z(0)=0.01$, showing the time variation in each compartment of the model with varying values of $e$ as shown in the legend (other parameters are set as in (11) except $n=0.16$ ). The simulations are carried out for two cases of infection rate, (a) low infection ( $a=0.1)$ and (b) high infection $(a=0.5)$

\section{Conclusions}

In this paper, we modified the model in [10] so that the dynamics of virotherapy incorporates the interaction between uninfected tumor cells and immune response. We have validated the plausibility of the model by demonstrating positivity and boundedness of solutions. Moreover, the model was analyzed qualitatively using the stability theory of nonlinear systems. As a result, five equilibrium points were obtained: $E_{0}$, indicating success in treatment; $E_{1}$, for failure in treatment; $E_{2}$, for virus dominance; $E_{3}$, for immune dominance; and $E_{4}$, for coexistence of all state variables. Our model created a new equilibrium point $\left(E_{3}\right)$ different from those in [10]. The importance of this equilibrium point 

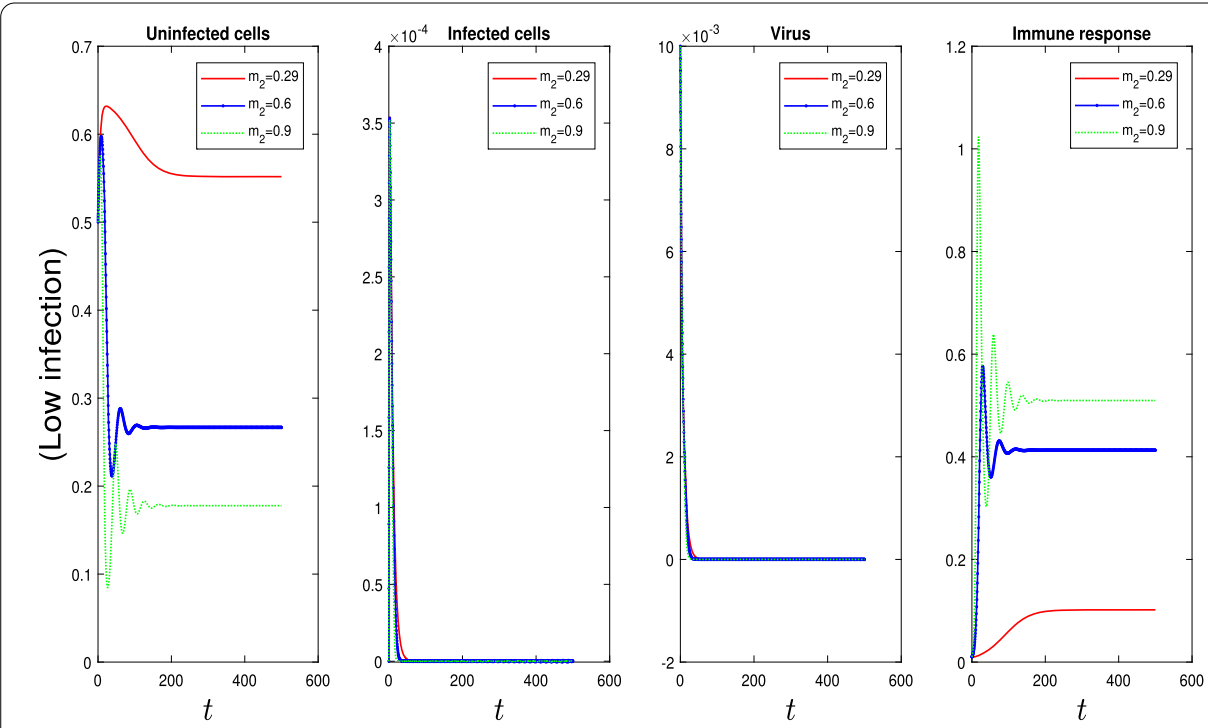

(a)
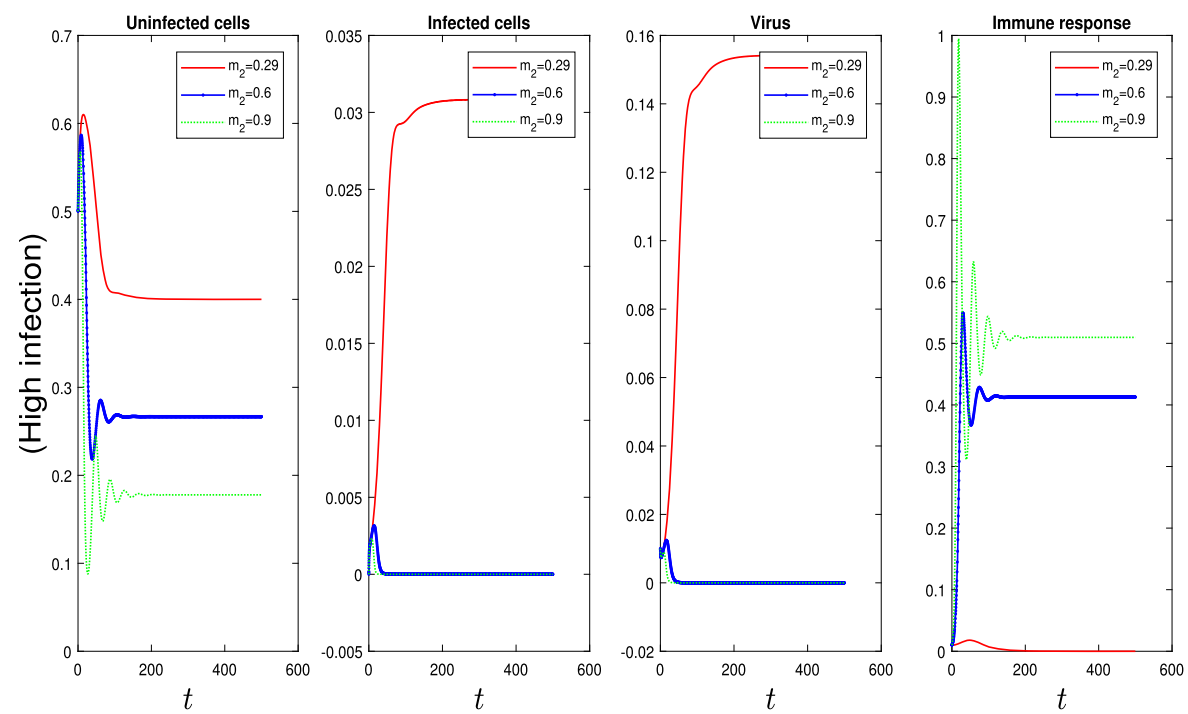

(b)

Figure 10 Numerical simulations of model (2) with initial values: $x(0)=0.5, y(0)=0, v(0)=0.01$, and $z(0)=0.01$, showing the time variation in each compartment of the model with varying values of $m_{2}$ as shown in the legend (other parameters are set as in (11) except $n=0.16)$. The simulations are carried out for two cases of infection rate, (a) low infection $(a=0.1)$ and $(\mathbf{b})$ high infection $(a=0.5)$

is that it shows the effect observed in virotherapy [2], where the immune system demolishes infected cells as well as viruses. The conditions of the existence and local stability of the equilibrium points have been determined. Furthermore, numerical experiments have shown the stability of the equilibrium points for different initial values. Thus, an agreement has been reached with qualitative results. Furthermore, a parameter analysis was performed on the following parameters: the burst size $(b)$, the relative immune killing rate of uninfected tumor cells $\left(h_{1}\right)$, the relative clearance rate of viruses $(e)$, and the relative stimulation rate of immune response by uninfected cells $\left(m_{2}\right)$. The analysis was carried out for two cases: low and high relative infection rates. As a result, partial success has 
been achieved in viral therapy provided that the burst size of the virus is large or that the rate of virus removal is low during high viral infection. Also, it was found that, during high viral infection, when the stimulation rate of immune response due to uninfected cells is low, the virus is prevalent and when high, the immune response is dominant. Moreover, in both cases, the size of the primary tumor decreases to an equilibrium value. Finally, the model may be extended to include a combination of virotherapy with other treatments to achieve total success and eradication of tumor cells. Also, the spatial evolution of cancer cells may be considered in models for future work.

\author{
Acknowledgements \\ Not applicable. \\ Funding \\ Not applicable. \\ Availability of data and materials \\ Not applicable. \\ Ethics approval and consent to participate \\ Not applicable.
}

Competing interests

The authors declare that they have no competing interests.

Consent for publication

Not applicable.

\title{
Authors' contributions
}

Conceptualization, SA-T; Formal analysis, SA-T and NA-J; Investigation, NA-J; Methodology, NA-J and SA-T; Resources, ES; Software, ES; Supervision, SA-T and ES; Validation, ES; Writing —original draft, NA-J; Writing—review and editing, SA-T. All authors read and approved the final manuscript.

\section{Publisher's Note}

Springer Nature remains neutral with regard to jurisdictional claims in published maps and institutional affiliations.

Received: 8 June 2020 Accepted: 12 August 2020 Published online: 26 August 2020

\section{References}

1. Cancer (2018). http://www.who.int/news-room/fact-sheets/detail/cancer

2. Marelli, G., Howells, A., Lemoine, N.R., Wang, Y.: Oncolytic viral therapy and the immune system: a double-edged sword against cancer. Front. Immunol. 9, 866-873 (2018)

3. Wodarz, D.: Gene therapy for killing p53-negative cancer cells: use of replicating versus nonreplicating agents. Hum. Gene Ther. 14, 153-159 (2003)

4. Bajzer, Ž., Carr, T., Josić, K., Russell, S.J., Dingli, D.: Modeling of cancer virotherapy with recombinant measles viruses. J. Theor. Biol. 252, 109-122 (2008)

5. Tian, J.P.: The replicability of oncolytic virus: defining conditions in tumor virotherapy. Math. Biosci. Eng. 8, 841-860 (2011)

6. Ikawati, D.S.E., Kusumawinahyu, W.M., Trisilowati: A dynamical analysis on a tumour virotherapy model with standard incident rate. J. Trop. Life Sci. 7, 16-22 (2017)

7. Komarova, N.L., Wodarz, D.: Targeted Cancer Treatment in Silico. Birkhäuser, Basel (2014)

8. Wodarz, D.: Viruses as antitumor weapons: defining conditions for tumor remission. Cancer Res. 61, 3501-3507 (2001)

9. Ashyani, A., Mohammadinejad, H.M., Rabieimotlagh, O.: Hopf bifurcation analysis in a system for cancer virotherapy with effect of the immune system. Jordan J. Math. Stat. 9, 93-115 (2016)

10. Phan, T.A., Tian, J.P.: The role of the innate immune system in oncolytic virotherapy. Comput. Math. Methods Med. 2017, Article ID 6587258 (2017)

11. Al-Johani, N., Simbawa, E., Al-Tuwairqi, S:: Modeling the spatiotemporal dynamics of virotherapy and immune response as a treatment for cancer. Commun. Math. Biol. Neurosci. 2019, Article ID 28 (2019)

12. Simbawa, E., Al-Johani, N., Al-Tuwairqi, S.: Modeling the spatiotemporal dynamics of oncolytic viruses and radiotherapy as a treatment for cancer. Comput. Math. Methods Med. 2020, Article ID 3642654 (2020)

13. Fujie, K., Ito, A., Winkler, M., Yokota, T.: Stabilization in a chemotaxis model for tumor invasion. Discrete Contin. Dyn. Syst., Ser. A 36(1), 151-169 (2016)

14. Viglialoro, G., Woolley, T.E.: Boundedness in a parabolic-elliptic chemotaxis system with nonlinear diffusion and sensitivity and logistic source. Math. Methods Appl. Sci. 41(5), 1809-1824 (2018)

15. Li, T., Pintus, N., Viglialoro, G.: Properties of solutions to porous medium problems with different sources and boundary conditions. Z. Angew. Math. Phys. 70(3), 1-16 (2019) 
16. Wu, J., Lanier, L.L.: Natural killer cells and cancer. Adv. Cancer Res. 90, 127-156 (2003)

17. de Pillis, L.G., Radunskaya, A.E., Wiseman, C.L.: A validated mathematical model of cell-mediated immune response to tumor growth. Cancer Res. 65, 7950-7958 (2005)

18. Marcus, A., Gowen, B.G., Thompson, T.W., lannello, A., Ardolino, M., Deng, W., Wang, L., Shifrin, N., Raulet, D.H. Recognition of tumors by the innate immune system and natural killer cells. Adv. Immunol. 122, 91-128 (2014)

19. Moynihan, K.D., Irvine, D.J.: Roles for innate immunity in combination immunotherapies. Cancer Res. 77, 5215-5221 (2017)

20. Lowry, L.E., Zehring, W.A.: Potentiation of natural killer cells for cancer immunotherapy: a review of literature. Front. Immunol. 8, 1061 (2017)

21. van den Driessche, P., Watmough, J.: Reproduction numbers and sub-threshold endemic equilibria for compartmental models of disease transmission. Math. Biosci. 180, 29-48 (2002)

22. Edelstein-Keshet, L.: Mathematical Model in Biology. Society for Industrial and Applied Mathematics, Philadelphia (2005)

23. Martcheva, M.: An Introduction to Mathematical Epidemiology, vol. 61. Springer, Berlin (2015)

24. Friedman, A., Tian, J.P., Fulci, G., Chiocca, E.A., Wang, J.: Glioma virotherapy: effects of innate immune suppression and increased viral replication capacity. Cancer Res. 66, 2314-2319 (2006)

25. Titze, M.I., Frank, J., Ehrhardt, M., Smola, S., Graf, N., Lehr, T.: A generic viral dynamic model to systematically characterize the interaction between oncolytic virus kinetics and tumor growth. Eur. J. Pharm. Sci. 97, 38-46 (2017)

26. Tao, Y., Guo, Q.: A free boundary problem modelling cancer radiovirotherapy. Math. Models Methods Appl. Sci. 17, $1241-1259(2007)$

27. Shampine, L., Reichelt, M.: The Matlab ODE suite. J. Sci. Comput. 18, 1-22 (1997)

28. Li, T., Rogovchenko, Y.V.: On the asymptotic behavior of solutions to a class of third-order nonlinear neutral differential equations. Appl. Math. Lett. 105, 106293 (2020)

\section{Submit your manuscript to a SpringerOpen ${ }^{\circ}$ journal and benefit from:}

- Convenient online submission

Rigorous peer review

- Open access: articles freely available online

- High visibility within the field

- Retaining the copyright to your article

Submit your next manuscript at $\gg$ springeropen.com 